\title{
Design and optimization of three-resonator locally resonant metamaterial for impact force mitigation
}

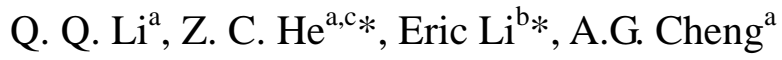 \\ a State Key Laboratory of Advanced Design and Manufacturing for Vehicle Body, Hunan University, \\ Changsha, $410082 \quad$ P. R. China \\ b Department of Mechanical and Automation Engineering, The Chinese University of Hong Kong, \\ Shatin, NT, Hong Kong, China \\ c The State Key Laboratory of Fluid Power and Mechatronic Systems, Zhejiang University, Hangzhou, \\ 310027, China
}

\begin{abstract}
Locally resonant metamaterials (LMs) have received extensive attention for their extraordinary physical properties, while less study on their application in the field of collision exists. In this paper, we extend the application of LMs to field of structural collision and achieve the mitigation of impact force based on their negative effective mass property. We also propose a three-resonator metamaterial (TRM) to enhance the attenuation effect of impact stress waves. Based on the theoretical analysis, the width of the negative effective mass frequency regions of TRM is wider than those of single-resonator metamaterial (SRM) and dual-resonator metamaterial (DRM). In numerical cases, the superior performance of TRM is also validated based on the impact wave model and the non-convex multi-corner thin-walled column crash model compared with SRM and DRM. The multi-objective optimization analyses are also conducted to enhance the performance of TRM with high impact force mitigation, great improvement of vehicle crashworthiness and small LMs mass.
\end{abstract}

Key words: locally resonant metamaterials; TRM; negative effective mass; impact force;

\footnotetext{
* Corresponding author.

E-mail address: hezhicheng815@163.com (Z. C. He); ericsg2012@gmail.com (Eric Li)
}

\section{Introduction}

Crashworthiness is a basic problem of vehicle safety, and numerous works on the 
vehicle crashworthiness [1, 2] have been carried out. As plastic deformation of structure in collision absorbs substantial kinetic energy, energy-absorbing components are widely used in body structural to absorb kinetic energy and reduce occupant damage. A lot of studies in energy-absorbing structures including foam filling [3-5], honeycomb filling [6] and negative Poisson's ratio material filling [7] have been explored in the past. Although many works on energy absorption structure have been carried out, the studies to reduce the impact force are still rare. As the larger impact force means higher vehicle acceleration and greater risk of occupant damage, it is important to develop novel materials that can mitigate this transient dynamic load. In addition, the propagation of stress waves during the collision causes the mechanical deformation and failure of structure. Therefore, the realization of the mitigation of stress wave is essential to attenuate the impact force and protect the structure.

As the man-made materials, LMs [8-15] have attracted broad interest from scholars in recent years for their special properties. Their properties are customized through special microstructures instead of chemically synthesized. The concept of LMs originates from the study of electromagnetic wave with negative permittivity, negative permeability and negative refractive index [16-19]. Liu et al. [20] firstly proposed the concept of locally resonant LMs that exhibits the band gaps at low frequency. The centimeter-sized local resonance composite structure exhibits effective negative elastic constants at certain frequency ranges. Norbert et al. [21] demonstrated the left-handed propagation property of a one-dimensional resonant LMs. Assouar et al. [22] studied two plate-type LMs that have the magical property of achieving the 
high sound transmission loss. Based on the numerical and experiment studies, Chen et al. [23] showed the characteristics of stress wave propagation in a sandwich beam structure with local oscillators. The location and range of the band gaps of this structure can be manipulated through adjusting the local resonant frequencies of the oscillators. Zhu et al. [24], Huang and Sun [25] presented the approaches to represent the dynamic behavior of elastic metamaterials. The accuracy of their methods was demonstrated by comparing their theoretical results with the results of finite element analyses. Zhang et al. [26] studied the sound transmission loss in the membrane-type LMs. The results indicated that the transmission loss depends strongly on the attaching mass and the membrane properties. Wang et al. [27-29] studied the band gaps, transmission properties and effective mass density of two-dimensional LMs with the characteristics of comblike profile, chiral comblike interlayer and viscoelastic, respectively.

In addition, recent studies showed that the LMs which exhibit the negative effective mass property can effectively attenuate the mechanical waves [30-35]. Therefore, the negative effective mass property is the key point to achieve the stress wave attenuation. Wang [36] proposed a new typical unit cell of LMs, which can be tuned to produce negative mass and negative modulus. Yao et al. [37] had realized the negative effective mass property of the spring mass system through experimental analysis, and the transmission property of mechanical wave in the low frequency range was examined. Li and Chan [38] developed and demonstrated the concept of simultaneous negative effective mass density and bulk modulus. Liu et al. [39] and 
Zhai et al. [40] presented the existence of double-negative LMs.

Although so many studies on LMs exist, there are few researches on the application of LMs to realize the impact load attenuation. In this paper, we apply the LMs to the field of structural collisions and apply the metamaterials to solve the problem of load attenuation that cannot be achieved with conventional materials.

It is worth noting that there are many ways to improve the performance of LMs. The design of microstructure of LMs and the application of active control theory are two commonly used methods. The active control system can tailor the characteristics of LMs based on the demand in practical application, and enlarging the frequency regions of negative effective mass to improve the attenuation effects of LMs [49, 50]. In addition, the design of microstructure changes the micro-characteristics of LMs, and the macroscopic properties of LMs can be improved through designing the microscopic characteristics of structure. In this paper, the design of microstructure of LMs is the main content of our work.

It is obvious that the negative effective mass property of LMs depends on their microstructure significantly. The single-resonator microstructure and dual-resonator microstructure were widely studied in the past [41, 42]. It is clear that the dual-resonator metamaterial (DRM) works better than the single-resonator metamaterial (SRM) for mechanical wave attenuation [43]. Obviously, the optimal design of microstructure can achieve a wide range of frequency attenuation, which is very effective for the mitigation of high frequency dynamic loads such as collision problems. In this paper, we propose a three-resonator metamaterial (TRM) under the 
principle of maximizing the band gap regions. The mathematical model shows TRM has wider negative effective mass frequency regions than SRM and DRM. Numerical simulations of the impact wave model and the non-convex multi-corner thin-walled column crash model demonstrate the superior performance of TRM to SRM and DRM in frequency spectrum attenuation and impact force mitigation. Furthermore, the multi-objective optimization is conducted to obtain the optimal parameters of TRM to minimize the LMs mass, body acceleration and impact force. The improvement of the crashworthiness of the trolley model is proved clearly through the application of TRM.

This paper is organized as follows: Theoretical studies of SRM, DRM and TRM are carried out in Section 2. In Sections 3 and 4, numerical examples of impact wave model and thin-walled structure crash model are studied to demonstrate the superior performance of TRM to SRM and DRM. In addition, the multi-objective optimization is carried out with this thin-walled crash model to improve its impact performance. Section 5 is mainly about the application of TRM on the trolley crash model. Finally, the conclusions are summarized in Section 6.

\section{Microstructure of LMs}

\subsection{Single-resonator and dual-resonator LMs}

In this work, we study how the negative effective mass property of the microstructures of metamaterials affects their attenuation effects. For the better explanation, the concept of negative effective mass based on the single-resonator and dual-resonator microstructures is firstly illustrated. Fig. 1 (a) shows the unit cell of 
SRM that is comprised of rigid masses $m_{1}, m_{2}$ and linear spring $k_{2}$. The displacements of the inner mass and outer mass are $u_{1}$ and $u_{2}$. A one-dimensional spring-mass lattice system consisting of the single-resonator microstructures is shown in Fig. 1(b). In this one-dimensional lattice system, the outer spring with connects each unit cell is $k_{1}$, and the motion equations of the $j$ th unit cell can be calculated as follows.

$$
\begin{aligned}
& m_{1} \ddot{u}_{1}^{(j)}=k_{1}\left(u_{1}^{(j-1)}+u_{1}^{(j+1)}-2 u_{1}^{(j)}\right)+k_{2}\left(u_{2}^{(j)}-u_{1}^{(j)}\right) \\
& m_{2} \ddot{u}_{2}^{(j)}=k_{2}\left(u_{1}^{(j)}-u_{2}^{(j)}\right)
\end{aligned}
$$

where $u_{\alpha}^{(j)}$ is the displacement of oscillator $\alpha(\alpha=1$ or 2$)$ in the $j$ th unit cell, $k_{1}$ is the outer spring with connects each unit cell. Based on Bloch theory, the harmonic waveform of the displacement for unit cell $(j+n)$ th must satisfy the following formula.

$$
u_{\alpha}^{(\mathrm{j}+\mathrm{n})}=\widehat{u}_{\alpha} e^{\mathrm{i}(\mathrm{qx}+\mathrm{nqa}-\mathrm{\omega t})}
$$

where $u_{\alpha}^{(j+n)}$ is the displacement of oscillator $\alpha$ in the $(j+n)$ th unit cell, $\omega$ is the angular frequency, $q$ is the wavenumber, $\widehat{u}_{\alpha}$ is the displacement amplitude of the oscillator $\alpha$ and $a$ is the lattice constant. We substitute Eq. (3) into Eqs. (1) and (2) to obtain the dispersion equation of the lattice system.

$$
\cos q a=1-\frac{\delta}{2 \theta} \frac{\left(\omega / \omega_{2}\right)^{2}\left[\left(\omega / \omega_{2}\right)^{2}-(1+\theta)\right]}{\left(\omega / \omega_{2}\right)^{2}-1}
$$

where $\omega_{2}=\sqrt{k / m_{2}}$ is the locally resonant frequency of oscillator $m_{2}$, and the mass ratio and spring stiffness ratio are $\theta=m_{2} / m_{1}$ and $\delta=k_{2} / k_{1}$. Due to the periodicity of the SRM, the attenuation effect of the SRM can be studied through studying the negative effective mass property of a single-resonator microstructure, and the effects of $k_{1}$ are not considered. When this single-resonator lattice system is regarded as the 
monoatomic lattice system, the effective mass of the single-resonator microstructure must satisfy the Eq. (5).

$$
m_{e f f}=\frac{2 k_{1}(1-\cos q a)}{\omega^{2}}
$$

Based on Eqs. (4) and (5), we can derive the control equation of dimensionless effective mass of the single-resonator microstructure that is the ratio of effective mass $m_{\mathrm{eff}}$ and static mass $m_{\mathrm{st}}$.

$$
\frac{m_{\mathrm{eff}}}{m_{\mathrm{st}}}=1+\frac{\theta}{1+\theta}\left[\frac{\left(\omega / \omega_{2}\right)^{2}}{1-\left(\omega / \omega_{2}\right)^{2}}\right]
$$

where $m_{\mathrm{st}}=m_{1}+m_{2}$ is total static mass of one microstructure. Then, the motion ratio of inner mass $m_{2}$ and outer mass $m_{l}$ is obtained as follows:

$$
\frac{\widehat{u}_{2}}{\widehat{u}_{1}}=\frac{1}{1-\left(\omega / \omega_{2}\right)^{2}}
$$

Plot of dimensionless frequency $\omega / \omega_{2}$ against dimensionless effective mass $m_{\mathrm{eff}} / m_{\mathrm{st}}$ is shown in Figs. 1(c). In Fig. 1(c), we take the parameter $\theta$ as 2. As the dimensionless frequency $\omega / \omega_{2}$ approaches 1 , a narrow band gap region where the effective mass becomes negative appears. It is clearly seen that the generation of negative effective mass is related to the motion mechanism of oscillators. In order to reveal this mechanism, the displacement ratio of $m_{2}$ and $m_{1}$ is shown in Fig. 1(d). From Fig. 1(d), when frequency $\omega$ is greater than $\omega_{2}$, the motions of $m_{1}$ and $m_{2}$ are out of phase, which implies that the mechanical wave of this frequency range cannot pass through the LMs. The wave energy is transferred and stored into the negative motions of the inner mass instead of propagating along the LMs. As a consequence, the negative mechanical motions block and reflect the incoming wave. Therefore, we call the 
negative effective mass frequency regions the stopbands.

As shown in Fig. 1(e), the unit cell of DRM that contains three masses can be seen as the coupling of two single-resonator unit cells. The rigid masses of the dual-resonator microstructure are $m_{1}, m_{2}$ and $m_{3}$, and their displacements are $u_{1}, u_{2}$ and $u_{3}$. The stiffness of each spring is $k_{1}, k_{2}$ and $k_{3}$. Analogous to single-resonator lattice system, the motion equations of the jth unit cell of DRM can be written as follows.

$$
\begin{aligned}
& m_{1} \ddot{u}_{1}^{(j)}=k_{1}\left(u_{1}^{(j-1)}+u_{1}^{(j+1)}-2 u_{1}^{(j)}\right)+k_{3}\left(u_{3}^{(j)}-u_{1}^{(j)}\right) \\
& m_{1} \ddot{u}_{2}^{(j)}=k_{2}\left(u_{3}^{(j)}-u_{2}^{(j)}\right) \\
& m_{3} \ddot{u}_{3}^{(j)}=k_{2}\left(u_{2}^{(j)}-2 u_{3}^{(j)}\right)+k_{3}\left(u_{1}^{(j)}-u_{3}^{(j)}\right)
\end{aligned}
$$

Based on the same principle, the dispersion relation of the one dimensional DRM can be acquired as follow Eq. (11).

$$
\cos q a=1-\frac{\delta_{2}\left(\omega / \omega_{2}\right)^{2}}{2 \theta_{2}}\left(1+\frac{\theta_{3}\left(1+\theta_{1}-\left(\omega / \omega_{2}\right)^{2}\right)}{\left[1-\left(\omega / \omega_{2}\right)^{2}\right]\left[1+\delta_{1}-\left(\delta_{1} / \theta_{1}\right)\left(\omega / \omega_{2}\right)^{2}\right]-\delta_{1}}\right)
$$

where $\omega_{2}=\sqrt{k_{2} / m_{2}}$ is the local resonance frequency of $m_{2}$. The stiffness and mass ratios are $\delta_{1}=k_{2} / k_{3}, \delta_{2}=k_{2} / k_{1}, \theta_{1}=m_{2} / m_{3}, \theta_{2}=m_{2} / m_{1}$ and $\theta_{3}=m_{3} / m_{1}$. Then, the effective mass of the dual-resonator microstructure can be obtained as follows.

$$
\frac{m_{e f f}}{m_{s t}}=\frac{\theta_{1}}{\theta_{1}+\theta_{2}+\theta_{1} \theta_{2}}+\frac{\theta_{2}}{\theta_{1}+\theta_{2}+\theta_{1} \theta_{2}}\left[\frac{1+\theta_{1}-\left(\omega / \omega_{2}\right)^{2}}{\left[1-\left(\omega / \omega_{2}\right)^{2}\right]\left[1+\delta_{1}-\left(\delta_{1} / \theta_{1}\right)\left(\omega / \omega_{2}\right)^{2}\right]-\delta_{1}}\right]
$$

The motion ratios of $m_{2}$ to $m_{1}$ and $m_{3}$ to $m_{1}$ are shown in Eqs. (13) and (14).

$$
\begin{aligned}
\frac{\widehat{u}_{2}}{\widehat{u}_{1}}= & \frac{1}{1-\left(\omega / \omega_{2}\right)^{2}\left(1+\delta_{1}+\delta_{1} / \theta_{1}-\left(\delta_{1} / \theta_{1}\right)\left(\omega / \omega_{2}\right)^{2}\right)} \\
\frac{\widehat{u}_{3}}{\widehat{u}_{1}}= & \left(\frac{\delta_{1}}{\theta_{1}}+\frac{1-\left(\omega / \omega_{2}\right)^{2}+\theta_{1}}{\theta_{1}\left(1+1 / \delta_{1}\right)\left(1-\left(\omega / \omega_{2}\right)^{2}\right)-\left(1-\left(\omega / \omega_{2}\right)^{2}\right)\left(\omega / \omega_{2}\right)^{2}-\theta_{1}}\right)\left(\frac{\omega}{\omega_{2}}\right)^{2} \\
& -\left(\frac{\omega}{\omega_{2}}\right)^{2} \frac{\delta_{1}}{\theta_{3}}+1
\end{aligned}
$$


Fig. 1(f) illustrates the plot of the dimensionless frequency $\omega / \omega_{2}$ against dimensionless effective mass $m_{\mathrm{eff}} / m_{\mathrm{st}}$. The parameters of mass and spring stiffness ratios $\theta_{1}, \theta_{3}$ and $\delta_{1}$ are assumed as $1,0.8$ and 0.2 . It is obvious that there are two band gaps in Fig. 1(f), which means that DRM has wide stopbands.

The displacement amplitude ratios of $m_{2}$ to $m_{1}$ and $m_{3}$ to $m_{1}$ are shown in Figs. $1(\mathrm{~g})$ and $(\mathrm{h})$. The parameters are selected as $\theta_{1}=1$ and $\delta_{1}=0.2$ in Fig. $1(\mathrm{~g})$ and $\theta_{1}=1$, $\theta_{3}=0.8$ and $\delta_{1}=0.2$ in Fig. 1(h). It is worth noting that both $u_{2}$ and $u_{3}$ are in phase with $u_{1}$ at frequency lower than the first resonant frequency. However, when the frequency increases and occupies the region between the first and the second resonant frequencies, $u_{3}$ is initially out of phase with $u_{1}$ and then gradually becomes in phase with $u_{1}$, and $u_{2}$ is always out of phase with $u_{1}$. When the frequency exceeds the second resonant frequency, $u_{3}$ is out of phase with $u_{1}$ and $u_{2}$ is always in phase with $u_{1}$. Therefore, by comparing Figs. 1 (g), (h) with Fig. 1 (f), it can be concluded that: (1) in the first band gap, $m_{2}$ and $m_{3}$ work within the same frequency range to block the incoming wave through their out of phase inertial forces; (2) in the second band gap, $m_{2}$ works at the region below the second resonant frequency and $m_{3}$ works at the range above the second resonant frequency to suppress the incoming wave.

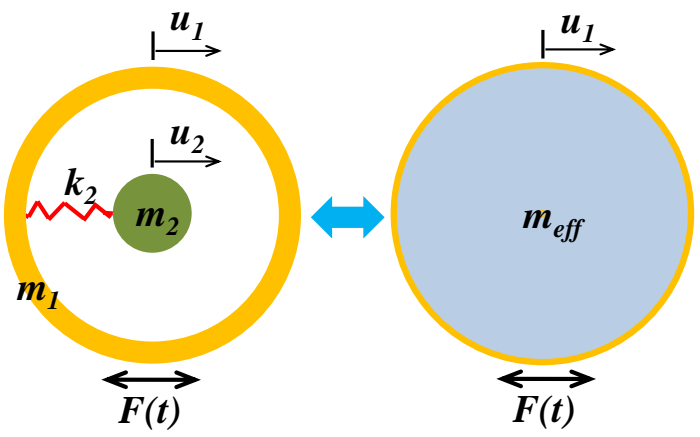

(a) 


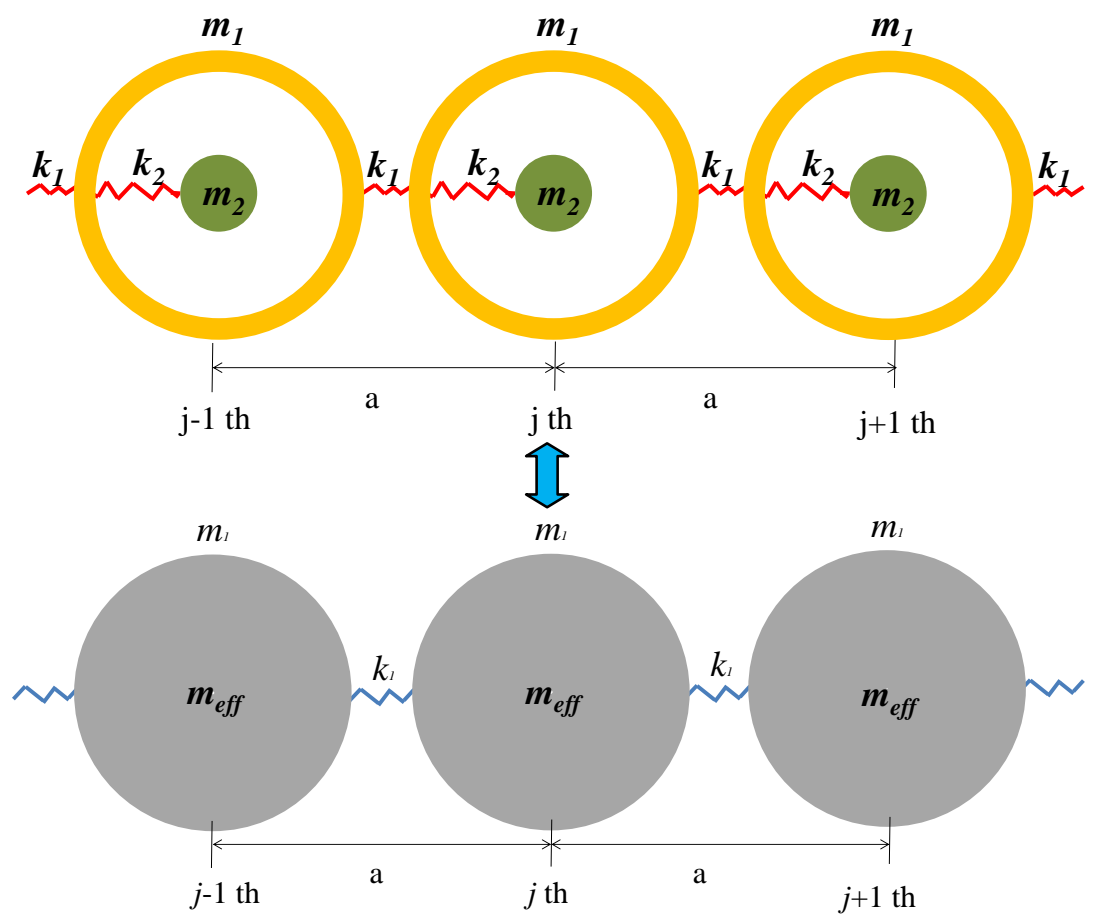

(b)

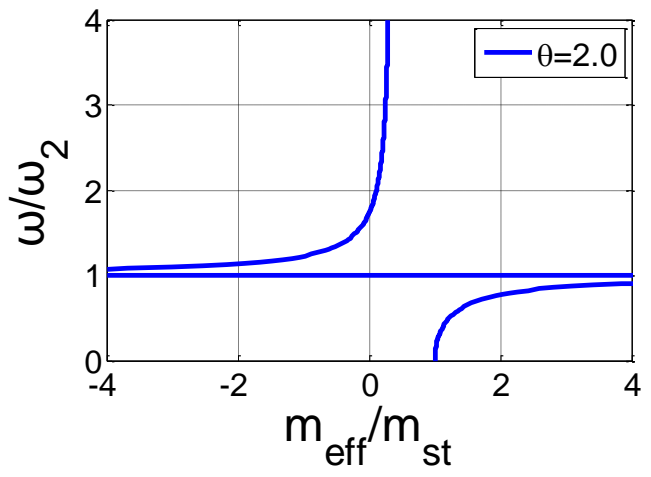

(c)

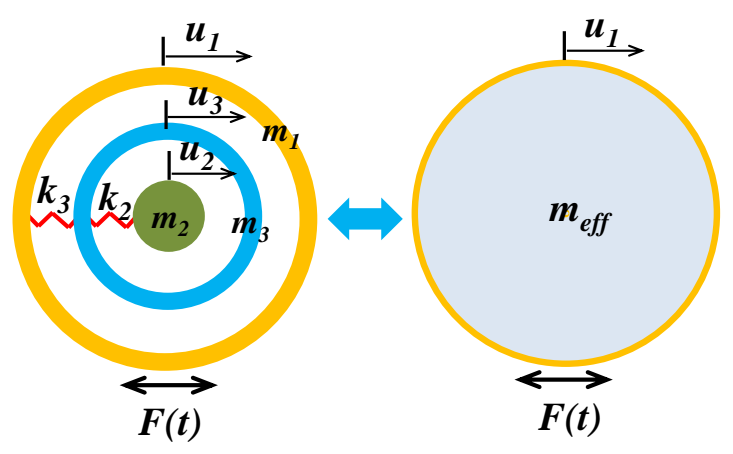

(e)

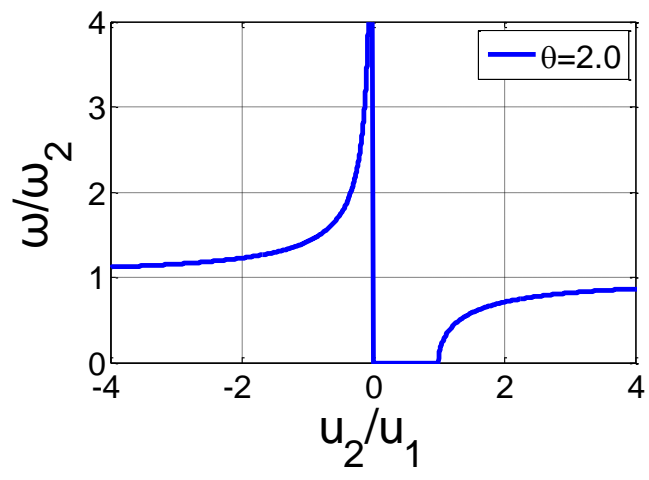

(d)

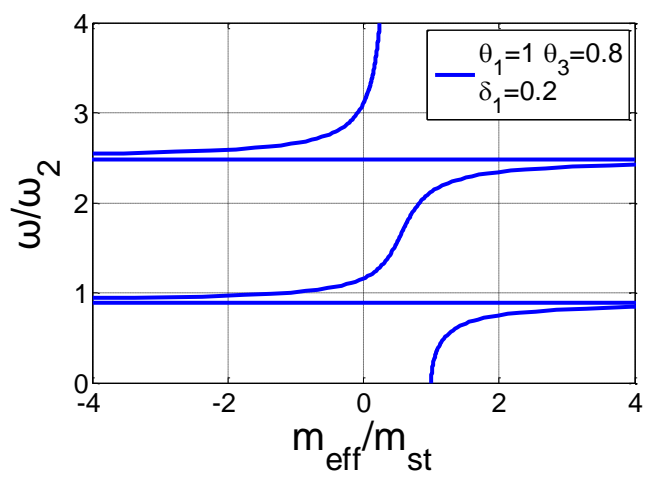

(f) 


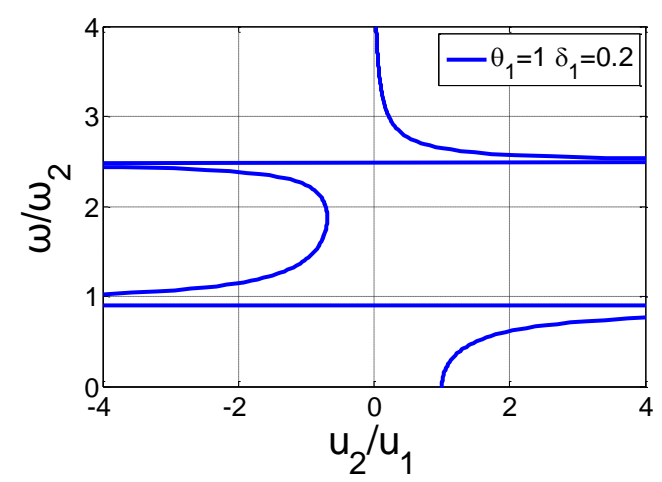

(g)

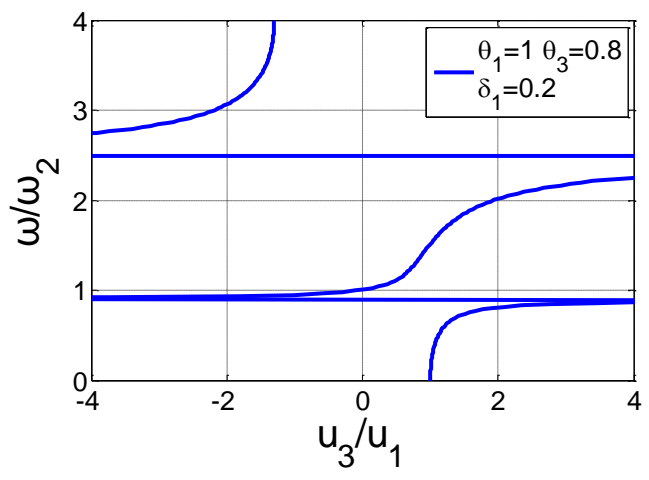

(h)

Fig. 1. (a) Microstructure of SRM. (b) One dimensional single-resonator lattice system. (c) Curve of dimensionless negative effective mass of SRM. (d) Curve of the dimensionless frequency $\omega / \omega_{2}$ against motion ratio $u_{2} / u_{1}$ of SRM with $\theta=2$. (e) Microstructure of DRM. (f) Curve of dimensionless negative effective mass of DRM with $\theta_{1}=1, \theta_{3}=0.8, \delta_{1}=0.2$. (g) Plot of dimensionless frequency $\omega / \omega_{2}$ against motion ratio $u_{2} / u_{1}$ of DRM with $\theta_{1}=1, \delta_{1}=0.2$. (h) Plot of dimensionless frequency $\omega / \omega_{2}$ against motion ratio $u_{3} / u_{1}$ of DRM with $\theta_{1}=1, \theta_{3}=0.8, \delta_{1}=0.2$.

\subsection{Three-resonator LMs}

As shown in Fig. 2(a), we propose a three-resonator microstructure that has four oscillators $m_{1}, m_{2}, m_{3}$ and $m_{4}$, and their movements are $u_{1}, u_{2}, u_{3}$ and $u_{4}$. The linear springs, which connect these rigid masses, are $k_{1}, k_{2}, k_{3}$ and $k_{4}$. Analogously, the control equations of oscillators of the $j$ th unit cell are calculated as follows.

$$
\begin{aligned}
& m_{1} \ddot{u}_{1}^{(j)}=k_{1}\left(u_{1}^{(j-1)}+u_{1}^{(j+1)}-2 u_{1}^{(j)}\right)+k_{4}\left(u_{4}^{(j)}-u_{1}^{(j)}\right) \\
& m_{2} \ddot{u}_{2}^{(j)}=k_{2}\left(u_{4}^{(j)}-u_{2}^{(j)}\right) \\
& m_{3} \ddot{u}_{3}^{(j)}=k_{3}\left(u_{4}^{(j)}-u_{3}^{(j)}\right) \\
& m_{4} \ddot{u}_{4}^{(j)}=k_{4}\left(u_{1}^{(j)}-u_{4}^{(j)}\right)+k_{3}\left(u_{3}^{(j)}-u_{4}^{(j)}\right)+k_{2}\left(u_{2}^{(j)}-u_{4}^{(j)}\right)
\end{aligned}
$$

The dispersion equation of the one dimensional TRM is obtained as Eq. (19). 


$$
\begin{aligned}
\cos q a=1-\frac{\beta_{2} \beta_{3}}{\beta_{3} r_{2} /\left(\omega / \omega_{2}\right)^{2}+\beta_{2} r_{3} /\left(\omega / \omega_{3}\right)^{2}}(1+ \\
\\
\\
\frac{r_{4}\left(1+\frac{\theta_{2}}{1-\left(\omega / \omega_{2}\right)^{2}}+\frac{\theta_{3}}{1-\left(\omega / \omega_{3}\right)^{2}}\right)}{1-\frac{\delta_{2}\left(\omega / \omega_{2}\right)^{2}}{2 \theta_{2}}-\frac{\delta_{3}\left(\omega / \omega_{3}\right)^{2}}{2 \theta_{3}}-\frac{\delta_{2}\left(\omega / \omega_{2}\right)^{2}}{1-\left(\omega / \omega_{2}\right)^{2}}-\frac{\delta_{3}\left(\omega / \omega_{3}\right)^{2}}{1-\left(\omega / \omega_{3}\right)^{2}}}
\end{aligned}
$$

where stiffness and mass ratios are $\beta_{2}=k_{2} / k_{1}, \beta_{2}=k_{3} / k_{1}, \gamma_{2}=m_{2} / m_{1}, \gamma_{3}=m_{3} / m_{1}, \gamma_{4}=$ $m_{4} / m_{1}, \delta_{2}=k_{2} / k_{4}, \delta_{3}=k_{3} / k_{4}, \theta_{2}=m_{2} / m_{4}$ and $\theta_{3}=m_{3} / m_{4}$. The effective mass $m_{\text {eff }}$ and dimensionless effective mass $m_{\mathrm{eff}} / m_{\mathrm{st}}$ of the new microstructure is acquired as Eqs. (20) and (21).

$$
\begin{aligned}
m_{\text {eff }} & =m_{1}+m_{4} \frac{1+\frac{\theta_{2}}{1-\left(\omega / \omega_{2}\right)^{2}}+\frac{\theta_{3}}{1-\left(\omega / \omega_{3}\right)^{2}}}{1-\frac{\delta_{2}\left(\omega / \omega_{2}\right)^{2}}{2 \theta_{2}}-\frac{\delta_{3}\left(\omega / \omega_{3}\right)^{2}}{2 \theta_{3}}-\frac{\delta_{2}\left(\omega / \omega_{2}\right)^{2}}{1-\left(\omega / \omega_{2}\right)^{2}}-\frac{\delta_{3}\left(\omega / \omega_{3}\right)^{2}}{1-\left(\omega / \omega_{3}\right)^{2}}} \\
& =m_{1}+m_{4} H
\end{aligned}
$$

$\frac{m_{\text {eff }}}{m_{\mathrm{st}}}=\frac{1}{1+\theta_{2} \gamma_{4}+\theta_{3} \gamma_{4}+\gamma_{4}}$

$$
+\frac{\gamma_{4}}{1+\theta_{2} \gamma_{4}+\theta_{3} \gamma_{4}+\gamma_{4}} \frac{1+\frac{\theta_{2}}{1-\left(\omega / \omega_{2}\right)^{2}}+\frac{\theta_{3}}{1-\left(\omega / \omega_{3}\right)^{2}}}{1-\frac{\delta_{2}\left(\omega / \omega_{2}\right)^{2}}{2 \theta_{2}}-\frac{\delta_{3}\left(\omega / \omega_{3}\right)^{2}}{2 \theta_{3}}-\frac{\delta_{2}\left(\omega / \omega_{2}\right)^{2}}{1-\left(\omega / \omega_{2}\right)^{2}}-\frac{\delta_{3}\left(\omega / \omega_{3}\right)^{2}}{1-\left(\omega / \omega_{3}\right)^{2}}}
$$

where $H$ stands for $\frac{1+\frac{\theta_{2}}{1-\left(\omega / \omega_{2}\right)^{2}}+\frac{\theta_{3}}{1-\left(\omega / \omega_{3}\right)^{2}}}{1-\frac{\delta_{2}\left(\omega / \omega_{2}\right)^{2}}{2 \theta_{2}}-\frac{\delta_{3}\left(\omega / \omega_{3}\right)^{2}}{2 \theta_{3}}-\frac{\delta_{2}\left(\omega / \omega_{2}\right)^{2}}{1-\left(\omega / \omega_{2}\right)^{2}}-\frac{\delta_{3}\left(\omega / \omega_{3}\right)^{2}}{1-\left(\omega / \omega_{3}\right)^{2}}}$.

The motion ratios of $m_{2}$ to $m_{1}, m_{3}$ to $m_{1}$ and $m_{4}$ to $m_{1}$ are acquired as follows.

$$
\begin{aligned}
& \frac{\widehat{u}_{2}}{\widehat{u}_{1}}=\frac{1}{1-\left(\omega / \omega_{2}\right)^{2}}+\frac{2 H \delta_{2}\left(\omega / \omega_{2}\right)^{2} \delta_{3}\left(\omega / \omega_{3}\right)^{2}}{\left(1-\left(\omega / \omega_{2}\right)^{2}\right)\left(\theta_{3} \delta_{2}\left(\omega / \omega_{2}\right)^{2}+\theta_{2} \delta_{3}\left(\omega / \omega_{3}\right)^{2}\right)} \\
& \frac{\widehat{u}_{3}}{\widehat{u}_{1}}=\frac{1}{1-\left(\omega / \omega_{3}\right)^{2}}+\frac{2 H \delta_{2}\left(\omega / \omega_{2}\right)^{2} \delta_{3}\left(\omega / \omega_{3}\right)^{2}}{\left(1-\left(\omega / \omega_{3}\right)^{2}\right)\left(\theta_{3} \delta_{2}\left(\omega / \omega_{2}\right)^{2}+\theta_{2} \delta_{3}\left(\omega / \omega_{3}\right)^{2}\right)}
\end{aligned}
$$




$$
\frac{\widehat{u}_{4}}{\widehat{u}_{1}}=\frac{1}{1-\frac{\delta_{2}}{2 \theta_{2}}\left(\frac{\omega}{\omega_{2}}\right)^{2}+\frac{\delta_{3}}{2 \theta_{3}}\left(\frac{\omega}{\omega_{3}}\right)^{2}-\delta_{2} \frac{\left(\omega / \omega_{2}\right)^{2}}{1-\left(\omega / \omega_{2}\right)^{2}}-\delta_{3} \frac{\left(\omega / \omega_{3}\right)^{2}}{1-\left(\omega / \omega_{3}\right)^{2}}}
$$

3D graphs of dimensionless effective mass $m_{\mathrm{eff}} / m_{\mathrm{st}}$ against dimensionless frequency $\omega / \omega_{2}$ and $\omega / \omega_{3}$ in global view is shown in Fig. 2(b). Three band gaps appear in the 3D graph, which implies TRM has larger negative effective mass frequency regions than SRM and DRM for its more band gaps. From Eq. (21), the value of $m_{\mathrm{eff}} / m_{\mathrm{st}}$ mainly depends on the design of parameters $\theta_{2}, \delta_{2}, \theta_{3}, \delta_{3}$ and $\gamma_{4}$. Attention is focused on the effects of these parameters on the locations and the widths of the multiple band gaps. Surface plots of $m_{\mathrm{eff}} / m_{\mathrm{st}}$ against $\omega / \omega_{2}$ and $\omega / \omega_{3}$ in bottom view are applied to study the TRM in detail. In Fig. 2 (c), the blank areas represent the stopband regions and the other areas are the passband regions. The mass and stiffness ratios are assumed as $\theta_{2}=0.8, \theta_{3}=0.8, \gamma_{4}=0.8, \delta_{2}=1$ and $\delta_{3}=1$. Because of the symmetry of the microstructure of TRM, parameters $\theta_{2}, \delta_{2}$ and $\theta_{3}, \delta_{3}$ have the same effects on the performance of TRM. Therefore, only parameters $\theta_{2}, \gamma_{4}$ and $\delta_{2}$ are studied. In Fig. 2 (c) and (d), it is clear that the stopband regions increase as $\theta_{2}$ changes from 0.8 to 5 , which means that $\theta_{2}$ has a positive effect on the negative effective mass frequency ranges. In addition, as $\theta_{2}$ increases, the stopband frequencies are also boosted in the $\omega / \omega_{2}$ axis. From Figs. 2 (c) and (e), the band gap regions are broadened as $\gamma_{4}$ changes to 8 from 0.8 . Furthermore, with the change of $\gamma_{4}$, their negative effective mass frequencies remain the same. Figs. 2 (c) and (f) show that small $\delta_{2}$ broadens these band gaps. The reduction of $\delta_{2}$ enhances the stopband frequencies in the $\omega / \omega_{2}$ axis. Therefore, the optimization of the stiffness and mass ratios of TRM is important to achieve the 
maximum stopband regions within the target frequency ranges.

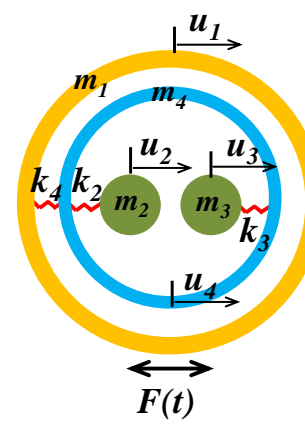

(a)

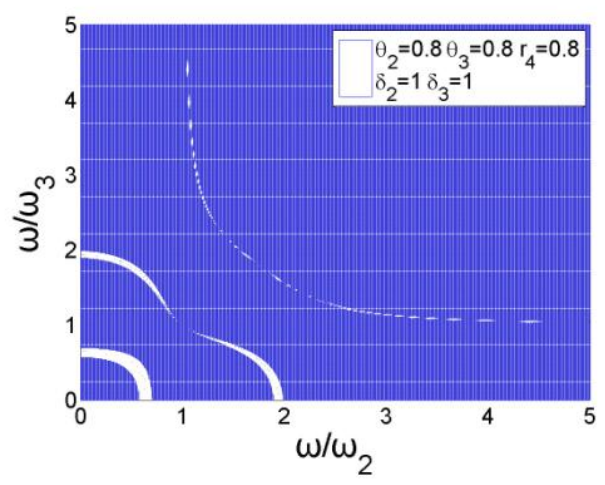

(c)

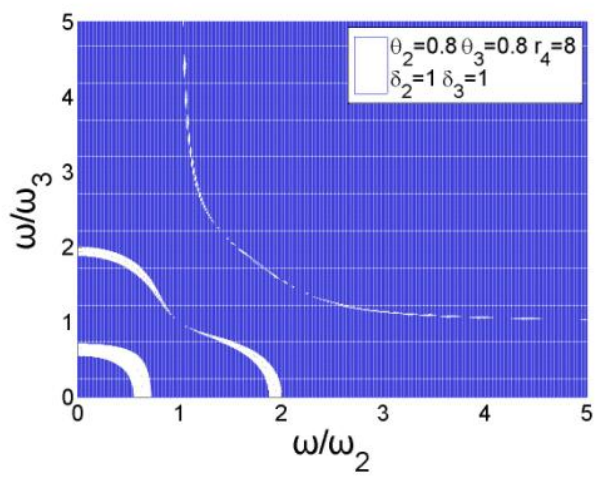

(e)

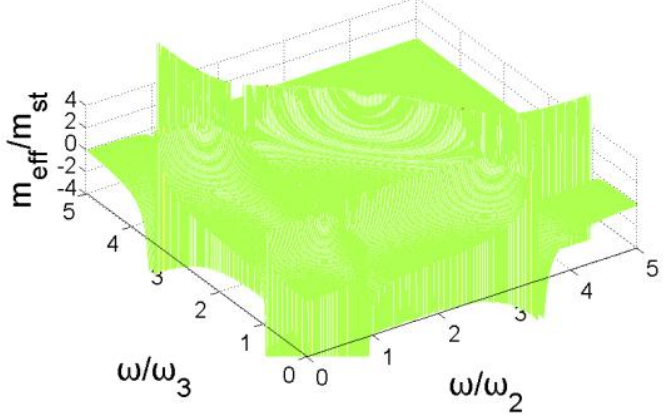

(b)

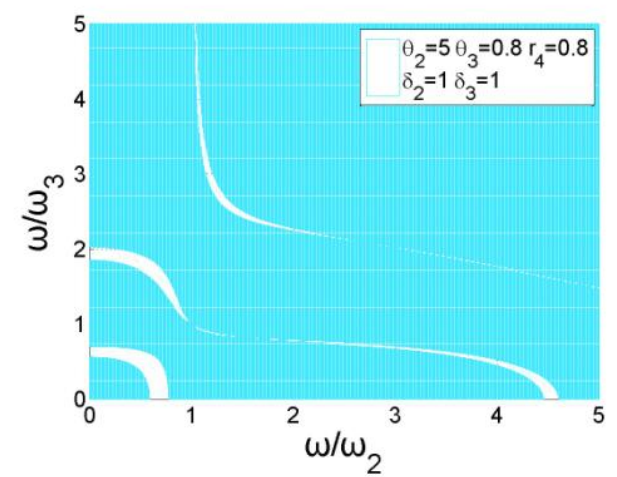

(d)

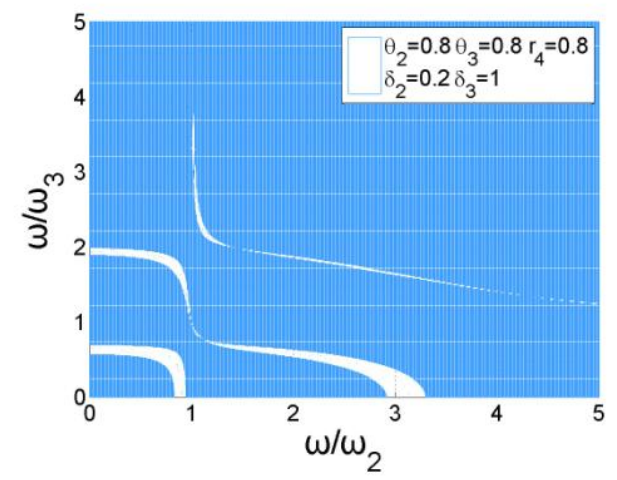

(f)

Fig. 2. (a) Microstructure of TRM. (b) 3D surface of dimensionless effective mass against dimensionless frequency of $\omega / \omega_{2}$ and $\omega / \omega_{3}$ in global view. (c) 3D surface of dimensionless effective mass against dimensionless frequency of $\omega / \omega_{2}$ and $\omega / \omega_{3}$ in bottom view with $\theta_{2}=0.8, \theta_{3}=0.8, \gamma_{4}=0.8, \delta_{2}=1, \delta_{3}=1$. (d) $\theta_{2}=5, \theta_{3}=0.8, \gamma_{4}=0.8, \delta_{2}=1$, $\delta_{3}=1$. (e) $\theta_{2}=0.8, \theta_{3}=0.8, \gamma_{4}=8, \delta_{2}=1, \delta_{3}=1$. (f) $\theta_{2}=0.8, \theta_{3}=0.8, \gamma_{4}=0.8, \delta_{2}=0.2, \delta_{3}=1$.

Since the displacement amplitude ratios of $\widehat{u}_{2} / \widehat{u}_{1}$ and $\widehat{u}_{3} / \widehat{u}_{1}$ are very similar, only the studies on $\hat{u}_{2} / \widehat{u}_{1}$ are carried out. Figs. 3 and 4 are the surface plots of 
motion ratios $\hat{u}_{4} / \widehat{u}_{1}$ and $\hat{u}_{2} / \widehat{u}_{1}$ against $\omega / \omega_{2}$ and $\omega / \omega_{3}$. Among them, Figs. 3 (a) and 4(a) are in the global view and the other figures are in the bottom view. When the frequency $\omega$ is in the range of the band gaps, the motions of the corresponding oscillators are out of phase. In other domain, their motions are in phase.

In Figs. 3 (a)-(f), it is clear that the distribution of the band gap regions of $\hat{u}_{4} / \hat{u}_{1}$ is similar to that of the negative effective mass $m_{\mathrm{eff}} / m_{\mathrm{st}}$. It means that when the negative effective mass appears, $u_{4}$ is always out of phase with $u_{1}$. The motion of $m_{4}$ always enhances the stopbands. As $\theta_{2}$ is increased from 0.8 to 4.0 in Figs. 3(b) and (c), and $\delta_{2}$ is reduced from 1.0 to 0.2 in Figs. 4 (b) and (d), the significant increase of band gap regions is achieved. These indicate that the enhancement of mass ratios and the reduction of stiffness ratios are in favor of the reverse motion of the inner mass $m_{4}$ to the outer mass $m_{1}$.

As shown in Figs. 4, the band gaps of $\widehat{u}_{2} / \widehat{u}_{1}$ are quite different from that of $m_{\mathrm{eff}} / m_{\mathrm{st}}$, which means that the motion of $m_{2}$ is complicated compared with $m_{4}$. In Fig. 3 (b) and Fig. 4 (b), when $\omega / \omega_{2}=0.5$ and $\omega / \omega_{3}=0.5$, the motions of $m_{2}$ and $m_{4}$ are all out of phase with $m_{1} . m_{2}$ and $m_{4}$ work together to block the incoming wave through their out of phase inertial forces. When $\omega / \omega_{2}=1$ and $\omega / \omega_{3}=4$, the motion of $m_{2}$ is out of phase with $m_{1}$ and $m_{4}$ is in phase with $m_{1}$. Only $m_{2}$ works to suppress the incoming wave through its own inertia force. When $\omega / \omega_{2}=2$ and $\omega / \omega_{3}=0$, the motion of $m_{2}$ is in phase with $m_{1}$ and $m_{4}$ is out of phase with $m_{1}$. Only $m_{4}$ works to compress the incident wave.

Despites these different band gap regions and motion behavior of $m_{2}$ and $m_{4}$, the 
parameter analyses show their similar results achieved by changing the same parameters. The broad band gaps of $\hat{u}_{2} / \widehat{u}_{1}$ can be observed when $\theta_{2}$ and $\theta_{3}$ are increased from 0.8 to 4.0 in Figs. 4(b) - (d), and $\delta_{2}$ and $\delta_{4}$ are decreased from 1.0 to 0.2 in Figs. 4(b), (e) and (f). The effect of $\hat{u}_{4} / \hat{u}_{1}$ on the band gaps is the same with $\widehat{u}_{2} / \widehat{u}_{1}$. The increase of mass ratios and the reduction of stiffness ratios are beneficial to the reverse motion of $m_{2}$ and $m_{1}$, which is effective to create the negative mass. These studies conducted in this section can provide a useful guidance to achieve wide band gaps in the desired frequency regions.

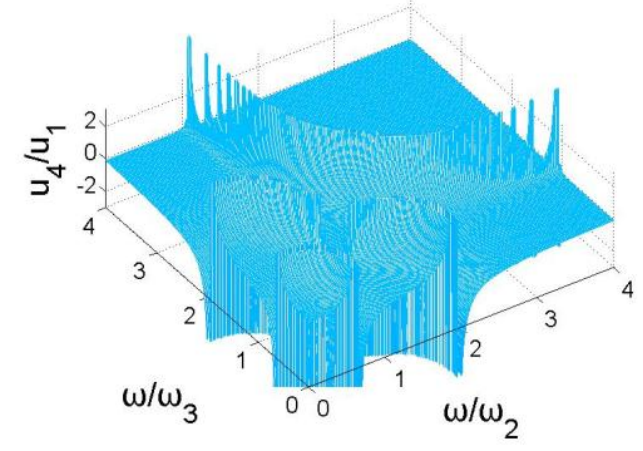

(a)

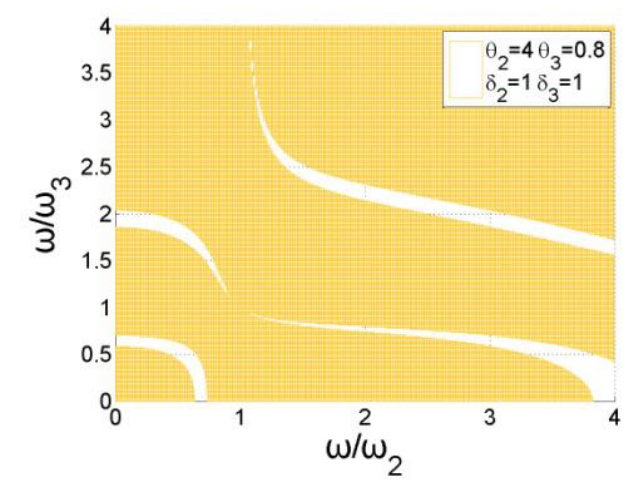

(c)

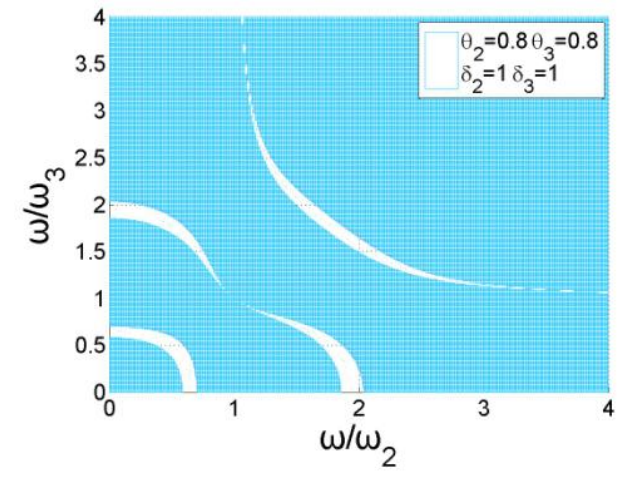

(b)

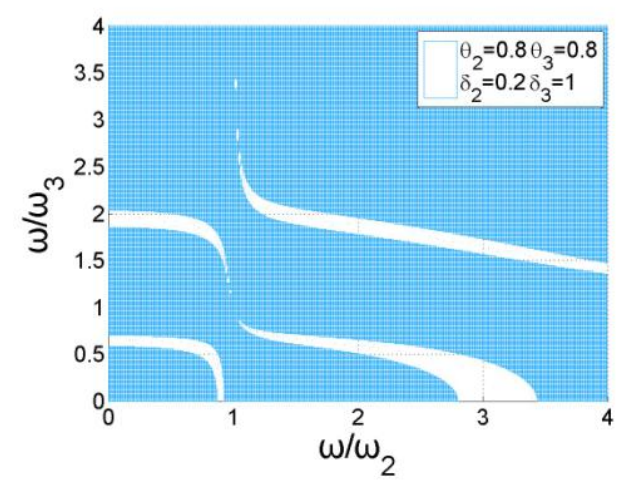

(d)

Fig. 3. (a) 3D surface of motion ratio of $u_{4} / u_{1}$ against dimensionless frequency of $\omega / \omega_{2}$ and $\omega / \omega_{3}$ in global view. (b) 3D surface of motion ratio of $u_{4} / u_{1}$ against dimensionless frequency of $\omega / \omega_{2}$ and $\omega / \omega_{3}$ in bottom view with $\theta_{2}=0.8, \theta_{3}=0.8$, $\delta_{2}=1, \delta_{3}=1$. (c) $\theta_{2}=4, \theta_{3}=0.8, \delta_{2}=1, \delta_{3}=1$. (d) $\theta_{2}=0.8, \theta_{3}=0.8, \delta_{2}=0.2, \delta_{3}=1$. 


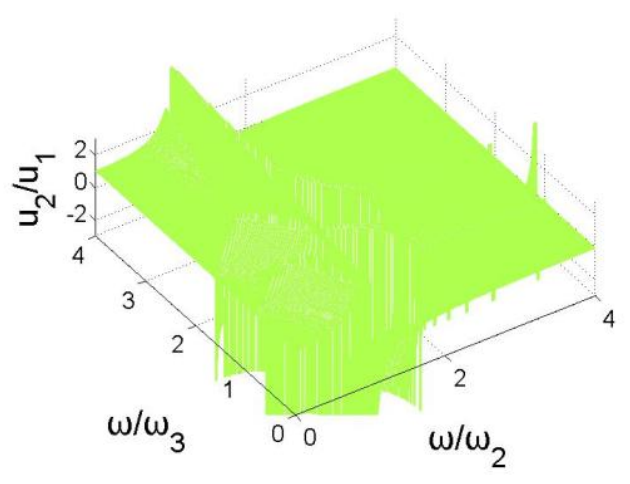

(a)

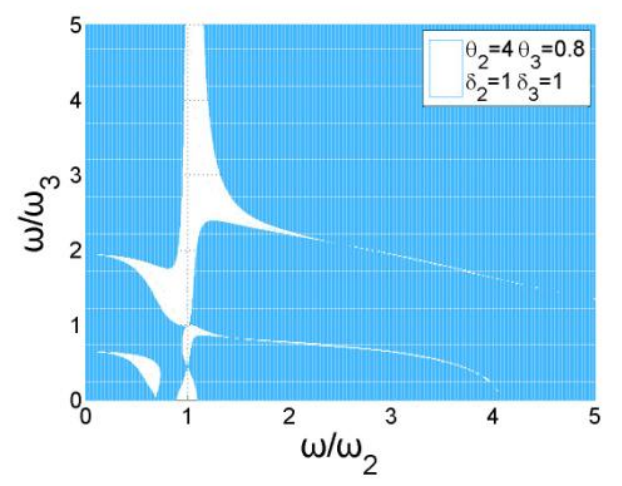

(c)

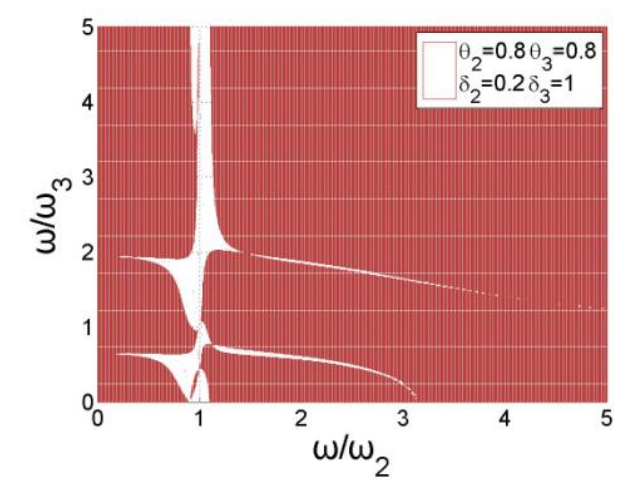

(e)

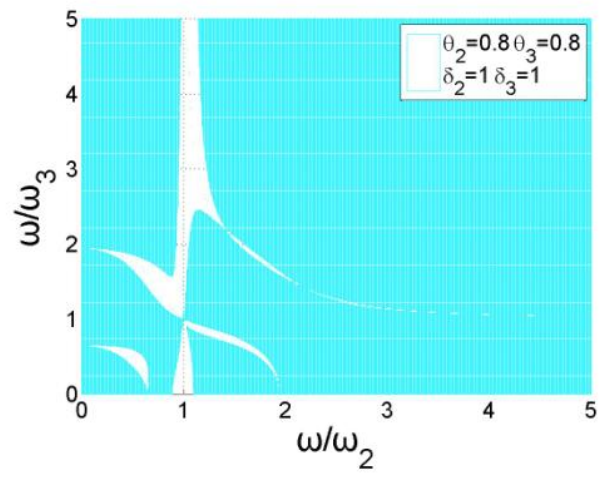

(b)

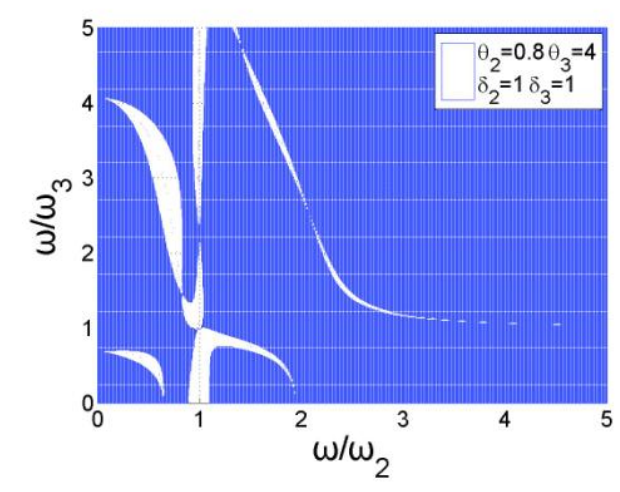

(d)

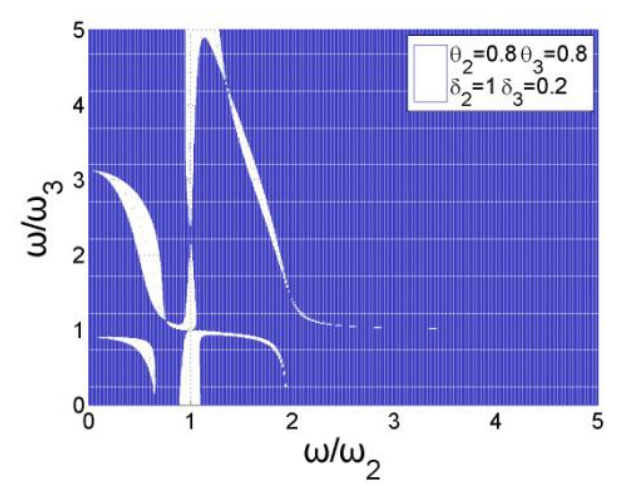

(f)

Fig. 4. (a) 3D surface of motion ratio of $u_{2} / u_{1}$ against dimensionless frequency of $\omega / \omega_{2}$ and $\omega / \omega_{3}$ in global view. (b) $3 \mathrm{D}$ surface of motion ratio of $u_{2} / u_{1}$ against dimensionless frequency of $\omega / \omega_{2}$ and $\omega / \omega_{3}$ in bottom view with $\theta_{2}=0.8, \theta_{3}=0.8$, $\delta_{2}=1, \delta_{3}=1$. (c) $\theta_{2}=4, \theta_{3}=0.8, \delta_{2}=1, \delta_{3}=1$. (d) $\theta_{2}=0.8, \theta_{3}=4, \delta_{2}=1, \delta_{3}=1$. (e) $\theta_{2}=0.8$, $\theta_{3}=0.8, \delta_{2}=0.2, \delta_{3}=1$. (f) $\theta_{2}=0.8, \theta_{3}=0.8, \delta_{2}=1, \delta_{3}=0.2$. 


\section{The impact wave model}

\subsection{The mitigation indicators of impact force}

In order to comprehensively assess the attenuation effect of LMs, it is necessary to define the evaluation indicators. Refer to the crashworthiness indicators studied in previous thin-wall crashworthiness researches [44, 45], the similar impact force attenuation indexes for LMs are proposed in this paper. These concepts include the peak impact force $(P I F)$ and the mitigation impact force $(M I F)$. The $M I F$ is defined as follows.

$$
M I F=P I F_{\mathrm{O}}-P I F_{\mathrm{A}}
$$

where $P I F_{\mathrm{O}}$ is the peak impact force of the original model and $P I F_{\mathrm{A}}$ is the peak impact force of the LMs model. A large MIF means a great impact force mitigation achieved by the LMs. The target is to maximize the MIF to protect the structure behind the LMs. At the same time, the LMs mass should also be minimized to achieve lightweight design of LMs. However, as we all know, great attenuation requires large LMs mass. Then, the specific impact force mitigation $(S F M)$ is defined as the attenuation of impact force per unit mass of the LMs to explain the relationship between the impact force mitigation and the LMs mass. It can be written as follows.

$$
S F M=M I F / M
$$

where $M$ is the total static mass of the LMs.

\subsection{Impact with one dimensional model}

We further study the attenuation effect of SRM, DRM and TRM with the impact 
wave model. The impact model contains a total of 400 unit cells, the first 100 unit cells is the Medium 1, the rear 290 unit cells (from unit cell \#111 to \#400) is the Medium 2 and the remaining 10 unit cells are applied to install the LMs (internal mass and outer mass for all LMs are $0.03 \mathrm{~kg}$ ). The force-controlled impact wave of $F=\widehat{F} e^{-\left(\mathrm{t}_{\mathrm{o}}\right) / \mathrm{t}_{\mathrm{d}}}\left(t_{0}=0.5 \mathrm{~ms}, t_{\mathrm{d}}=0.1 \mathrm{~ms}\right)$ with the duration time $4 \mathrm{~ms}$, is applied to the unit cell \#1. We acquire the impact force response at the unit cell \#130.

The results of SRM, DRM and TRM are shown in Fig. 5. Since one unit cell of TRM can be seen as the combination of one unit cell of SRM and one unit cell of DRM, the comparative study of 1-DRM: $(1.184,2.665) * 1 \mathrm{kN} / \mathrm{mm}+1$-SRM: $(0.296) * 1 \mathrm{kN} / \mathrm{mm}$ and 1-TRM: $(0.296,1.184,2.665)^{*} 1 \mathrm{kN} / \mathrm{mm}$ is carried out. Notations of "1-DRM: $(1.184,2.665) * 1 \mathrm{kN} / \mathrm{mm}+1-\mathrm{SRM}:(0.296) * 1 \mathrm{kN} / \mathrm{mm}$ ” mean that this model is composed by one dual-resonator unit cell and one single-resonator unit cell. The spring stiffness of this dual-resonator unit cell are $k_{2}=1.184 \mathrm{kN} / \mathrm{m}$ and $k_{3}=2.665 \mathrm{kN} / \mathrm{m}$, and the spring stiffness for this single-resonator unit cell is $k_{2}=$ $0.296 \mathrm{kN} / \mathrm{m}$.

Fig. 5(a) presents the attenuation results of these two LMs. Both of these two models have three band gaps. Similar frequency attenuation regions are achieved by 1-DRM: $(1.184,2.665) * 1 \mathrm{kN} / \mathrm{mm}+1$-SRM: $(0.296)^{*} 1 \mathrm{kN} / \mathrm{mm}$ and 1-TRM: $(0.296$, $1.184,2.665)^{*} 1 \mathrm{kN} / \mathrm{mm}$. It means that these two LMs have similar attenuation effects. From Fig. 5(b), the maximum impact force of the original model is 303N. Drop of peak impact force is achieved for these two LMs. The maximum impact force of 1-DRM: $(1.184,2.665)^{*} 1 \mathrm{kN} / \mathrm{mm}+1$-SRM: $(0.296)^{*} 1 \mathrm{kN} / \mathrm{mm}$ and 1-TRM: $(0.296$, 
$1.184,2.665)^{*} 1 \mathrm{kN} / \mathrm{mm}$ are $283.0 \mathrm{~N}$ and $284.5 \mathrm{~N}$, in which $S F M$ value are $133.3 \mathrm{~N} / \mathrm{kg}$ and $154.2 \mathrm{~N} / \mathrm{kg}$. Together with Fig. 5 (a), it is clear that the frequency spectrum attenuation and the impact force mitigation of these two LMs are close. However, the higher SFM value of 1-TRM: $(0.296,1.184,2.665) * 1 \mathrm{kN} / \mathrm{mm}$ compared with 1-DRM: $(1.184,2.665) * 1 \mathrm{kN} / \mathrm{mm}+1-\mathrm{SRM}:(0.296) * 1 \mathrm{kN} / \mathrm{mm}$ proves that TRM has better efficiency than SRM and DRM. This also implies that the TRM will certainly be thin and light. Mass and volume savings of TRM are mainly due to the lesser use of unit cells.

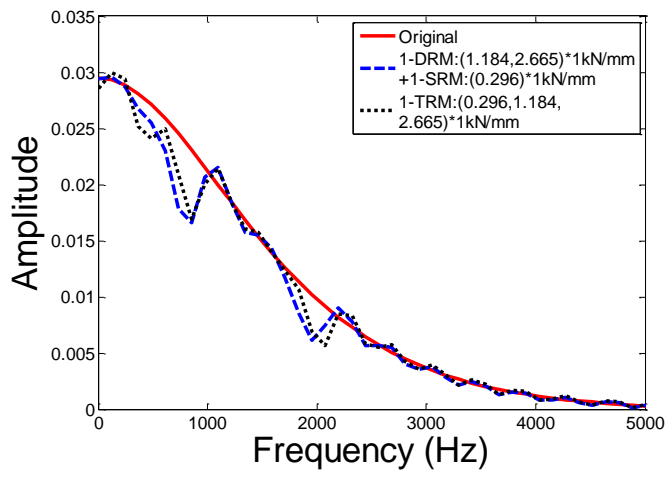

(a)

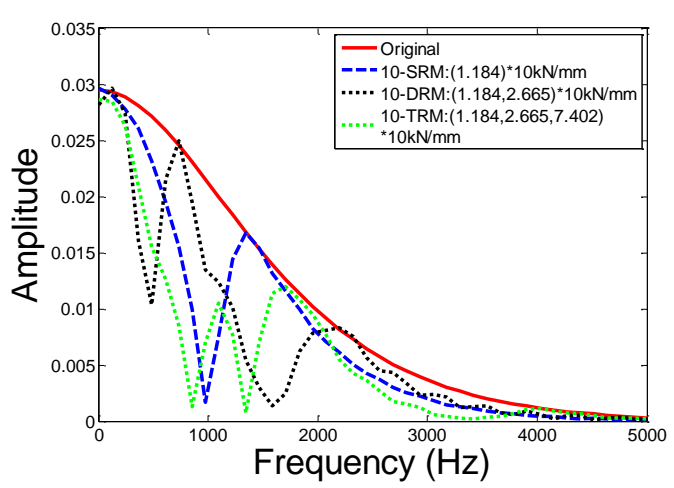

(c)

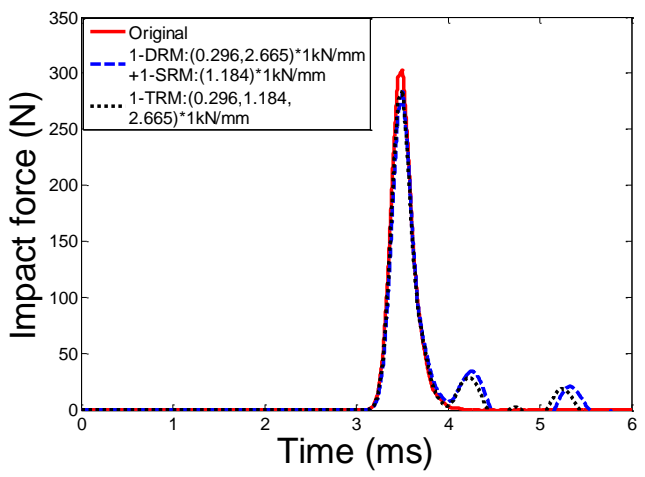

(b)

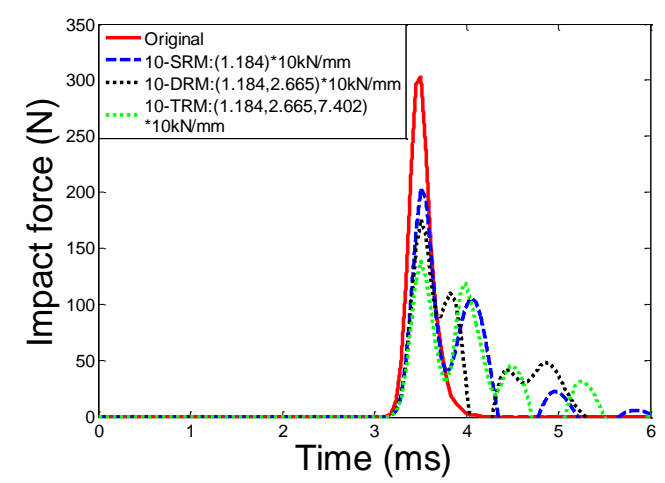

(d)

Fig. 5. (a) Attenuation of frequency spectrum with one DRM + one SRM unit cells and one TRM unit cell. (b) Mitigation of impact force with one DRM + one SRM unit cells and one TRM unit cell. (c) Attenuation of frequency spectrum with ten SRM unit cells, ten DRM unit cells and ten TRM unit cells. (d) Mitigation of impact force with ten SRM unit cells, ten DRM unit cells and ten TRM unit cells.

As shown in Figs. 5(c) and (d), the attenuation effect of SRM, DRM and TRM 
with the same ten unit cells is studied. From Figs. 5(c), 10-SRM: (1.184)*10 kN/mm has one band gap, 10-DRM: $(1.184,2.665) * 10 \mathrm{kN} / \mathrm{mm}$ has two attenuation regions of frequency spectrum, while 10-TRM: $(1.184,2.665,7.402)^{*} 10 \mathrm{kN} / \mathrm{mm}$ has three stopbands. It is clear that the frequency spectrum attenuation regions of 10-TRM: $(1.184,2.665,7.402)^{*} 10 \mathrm{kN} / \mathrm{mm}$ is the largest. As can be seen from Fig. 5(d), the maximum impact force of 10-SRM: $(1.184) * 10 \mathrm{kN} / \mathrm{mm}, 10-\mathrm{DRM}:(1.184,2.665) * 10$ $\mathrm{kN} / \mathrm{mm}$ and 10-TRM: $(1.184,2.665,7.402) * 10 \mathrm{kN} / \mathrm{mm}$ are $203 \mathrm{~N}, 176 \mathrm{~N}$ and $138 \mathrm{~N}$. With the same volume, the better performance of TRM is reflected.

\section{The thin-walled column impact model}

In this section, we study the attenuation effect of LMs on the practical crash condition. As shown in Fig. 6(a), the non-convex multi-corner thin-walled column structure [46], which is fully constrained at one end, is applied in the crash model. The materials of part 1 and part 2 are AL, that Young's modulus, Poisson's ratio and density are $2700 \mathrm{Mpa}, 0.3$ and $2.7 \mathrm{e}-6 \mathrm{~kg} / \mathrm{mm}^{3}$. The material of part 3 is steel, that material constants are $21000 \mathrm{Mpa}, 0.3$ and $7.85 \mathrm{e}-6 \mathrm{~kg} / \mathrm{mm}^{3}$ for Young's modulus, Poisson's ratio and density. The thickness of part 1, part 2 and part 3 is $1.0 \mathrm{~mm}$. As the deformation of the thin-walled column structure during the collision is small, it can be seen as the elastic deformation. Therefore, the material nonlinearity is not considered in this model. A rigid plate that mass is $1.6 \mathrm{~kg}$ vertical impacts this thin-walled structure with the initial speed of $50 \mathrm{~km} / \mathrm{s}$. Three types of LMs are applied in part 3, respectively. We obtain the transient dynamic load of the structure. 


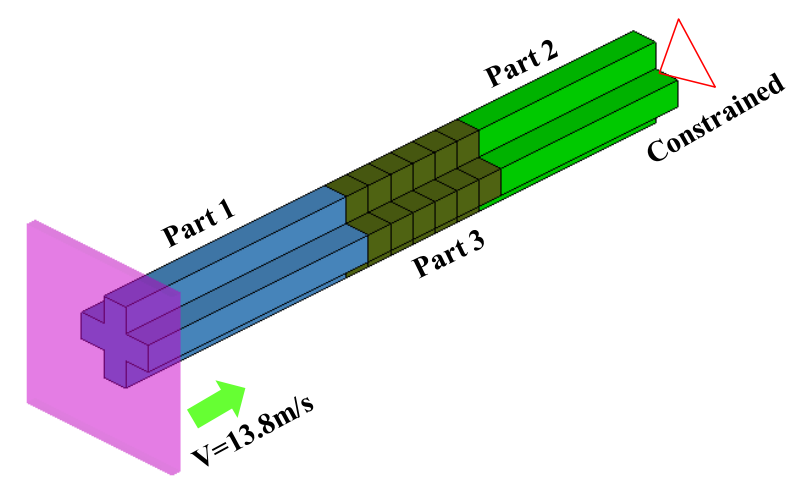

(a)

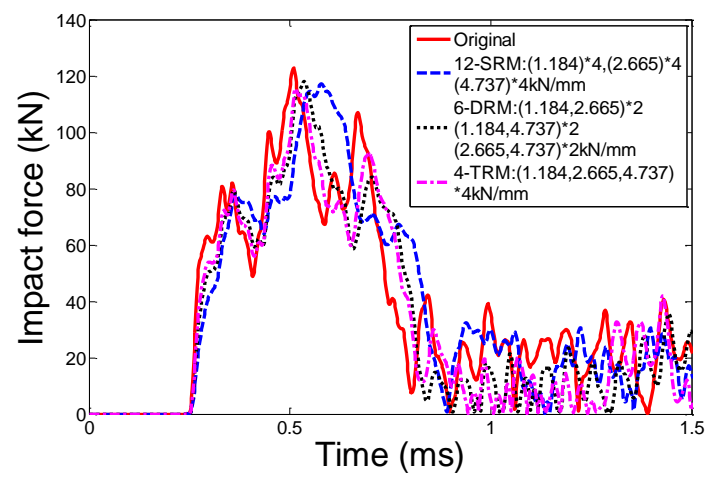

(b)

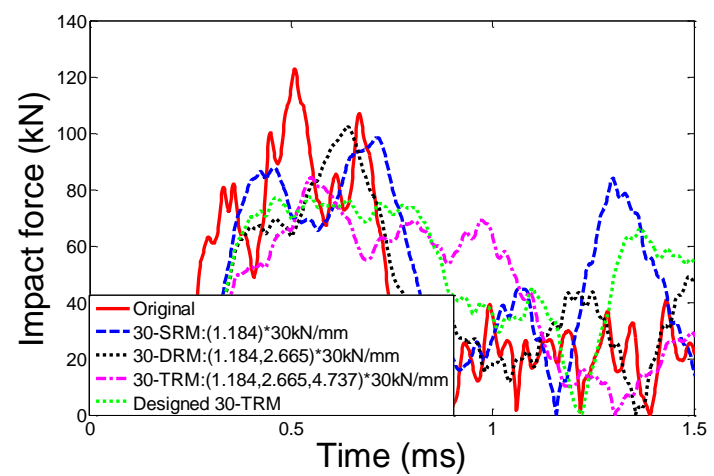

(c)

Fig. 6. (a) Multi-corner thin-walled column crash model with LMs. (b) Impact force attenuation for twelve SRM unit cells, six DRM unit cells, four TRM unit cells and ten TRM unit cells. (c) Impact force attenuation with thirty SRM, DRM, TRM unit cells and designed TRM.

For all LMs unit cells, the outer masses and internal oscillator masses are $0.01 \mathrm{~kg}$ and $0.03 \mathrm{~kg}$, respectively. Twelve SRM unit cells, six DRM unit cells and four TR unit cells are applied to further study the performance of TRM. Fig. 6(b) compares the results of 12-SRM: (1.184)*4, (2.665)*4, (4.737)*4 kN/mm, 6-DRM: (1.184, $2.665) * 2,(1.184,4.737)^{*} 2,(2.665,4.737) * 2 \mathrm{kN} / \mathrm{mm}$ and 4-TRM: $(1.184,2.665$, $4.737) * 4 \mathrm{kN} / \mathrm{mm}$. The maximum impact force of original model is $122.89 \mathrm{kN}$. Significant drop of impact force occurs for all LMs models from $0.3 \mathrm{~ms}$ to $1 \mathrm{~ms}$, and decrease of impact force implies the protection of this structure. Peak impact forces of SRM, DRM and TRM models are $117.28 \mathrm{kN}, 118.01 \mathrm{kN}$ and $115.51 \mathrm{kN}$, in which $S F M$ values are $11.69 \mathrm{kN} / \mathrm{kg}, 11.62 \mathrm{kN} / \mathrm{kg}$, and $18.45 \mathrm{kN} / \mathrm{kg}$. The superior 
performance of TRM to SRM and DRM is demonstrated for its higher SFM value and fewer unit cells. Fig. 6(c) illustrates the results of 30-SRM: (1.184)*30 kN/mm, 30-DRM: $(1.184,2.665) * 30 \mathrm{kN} / \mathrm{mm}$ and 30-TRM: $(1.184,2.665,4.737) * 30 \mathrm{kN} / \mathrm{mm}$. The maximum impact forces of SRM, DRM and TRM are $102.18 \mathrm{kN}, 98.50 \mathrm{kN}$ and 84.19 kN. With the same thirty unit cells, higher impact force attenuation is achieved by TRM.

The peak impact force, LMs mass and $S F M$ value of the 30-TRM: $(1.184,2.665$, 4.737)*30 kN/mm are $84.19 \mathrm{kN}, 2.7 \mathrm{~kg}$ and $14.33 \mathrm{kN} / \mathrm{kg}$. This high impact force mitigation and small $S F M$ value implies that a great attenuation effect requires a large LMs mass. However, the excessive mass limits the application of LMs on the real crash condition. Therefore, it is of great significance to maximize the impact force mitigation and minimize the LMs mass. The transient dynamic load mitigation behavior of TRM depends mainly on the design parameters of spring stiffness and oscillator mass. In this paper, we design the TRM by seeking the optimum combination of these parameters. The NSGA-II [47, 48] is applied as the optimization theory. Genetic algorithm (GA) is widely used in optimization problem that is based on Darwin's biological evolution and survival theory. The non-dominated sorting GA II (NSGA-II) [47, 48] is an extended algorithm for GA to solve multi-objective optimization problem, which was proposed in 2002. The success of the NSGA-II is largely due to the insertion of the concept of Pareto-optimality into the selection mechanism, and using the elite strategy and the crowding distance operator to rank the population. The crowding operator makes NSGA-II faster obtain the desired Pareto 
optimal solutions with less computational complexity. The optimization goals are to minimize the total static mass of LMs and maximize the MIF. In the optimization process, the outer mass $m_{1}$ of theses unit cells remains the same, the oscillator masses $m_{2}, m_{3}, m_{4}$ and spring stiffness $k_{2}, k_{3}, k_{4}$ are set as the design variables. The initial value and design space of each variable are shown in Table 1.

The whole optimization process is summarized as Fig. 7. In Fig. 7, the variables, design space and optimization goals are determined first based on the microstructure of TRM and our needs. Then, the parameterized model is established with TRM and this thin-walled crash structure. The multi-objective optimization analysis is carried out to obtain the optimal combination of spring stiffness and oscillator mass. After that, if the maximum iteration is achieved, optimization results will be verified by comparing with the 30 -TRM: $(1.184,2.665,4.737) * 30 \mathrm{kN} / \mathrm{mm}$ to determine whether these non-dominated solutions are reasonable. If so, the design of TRM ends. We will obtain the optimal solution based on our needs. Otherwise, re-optimize the TRM parameters again until the solutions meet the requirements. Finally, the designed TRM with high impact force mitigation and small LMs mass is acquired.

Table 1. The initial value and design space of each variable

\begin{tabular}{cccc}
\hline Design variable & Initial value & Lower & Upper \\
\hline$k_{2}(\mathrm{kN} / \mathrm{mm})$ & 1.184 & 0.098 & 8.71 \\
$k_{3}(\mathrm{kN} / \mathrm{mm})$ & 2.665 & 0.098 & 8.71 \\
$k_{4}(\mathrm{kN} / \mathrm{mm})$ & 4.737 & 0.89 & 12.34 \\
$m_{2}(\mathrm{~kg})$ & 0.03 & 0.01 & 0.05 \\
$m_{3}(\mathrm{~kg})$ & 0.03 & 0.01 & 0.05 \\
$m_{4}(\mathrm{~kg})$ & 0.03 & 0.01 & 0.05 \\
\hline
\end{tabular}




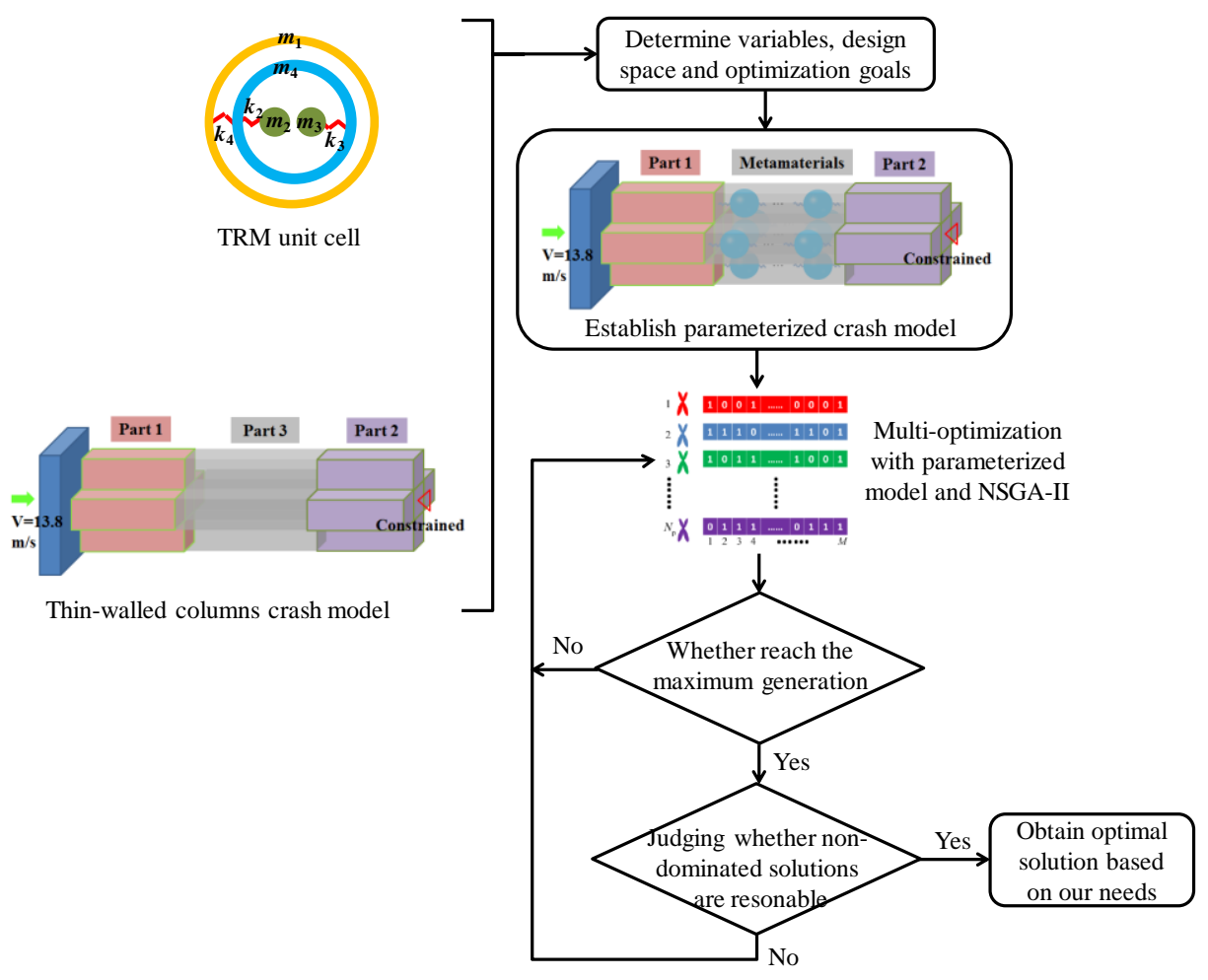

Fig. 7. Optimization process of the parameterized model

The mathematical model of the multi-objective optimization is described as follows:

$$
\begin{cases}\text { Min } & 1 / M I F, M \\ \text { s.t. } & 0.098<k_{2}<8.71 \mathrm{kN} / \mathrm{mm} \\ & 0.098<k_{3}<8.71 \mathrm{kN} / \mathrm{mm} \\ & 0.89<k_{4}<12.34 \mathrm{kN} / \mathrm{mm} \\ & 0.01<m_{2}<0.05 \mathrm{~kg} \\ & 0.01<m_{3}<0.05 \mathrm{~kg} \\ & 0.01<m_{4}<0.05 \mathrm{~kg}\end{cases}
$$

The Pareto frontier of the results of this model is illustrated in Fig. 8. As shown in Fig. 8, fifty feasible solutions in the design space are exhibited for us to select. Based on Eq. (26), the SFM of all solutions, in which the values are between 18.31 $\mathrm{kN} / \mathrm{kg}$ and $32.41 \mathrm{kN} / \mathrm{kg}$, are calculated. In order to better study these results, four non-dominated solutions, in which the largest and smallest values of SFM are included, are shown in Table 2. The results of $M I F, P I F_{\mathrm{E}}$ and $M$ are also listed. From 
Table 2, the option 1 should be selected if we prefer to obtain the maximum mitigation of impact force. If the mass of LMs is the critical factor, the option 4 is better than others. If the mass factors and the mitigation effect are equally important, options 2 and 3 should be considered. In this paper, we want to obtain a small peak impact force with small LMs mass. Therefore, the option 3 is selected. For this solution, parameters $k_{2}, k_{3}, k_{4}, m_{2}, m_{3}$ and $m_{4}$ are $3.37 \mathrm{kN} / \mathrm{mm}, 1.37 \mathrm{kN} / \mathrm{mm}, 1.85$ $\mathrm{kN} / \mathrm{mm}, 0.016 \mathrm{~kg}, 0.018 \mathrm{~kg}$ and $0.027 \mathrm{~kg}$. Compared with 30-TRM: (1.184, 2.665, 4.737)*30 kN/mm, the $S F M$ value of designed TRM increases from $14.33 \mathrm{kN} / \mathrm{kg}$ to $29.51 \mathrm{kN} / \mathrm{kg}$. In addition, Fig. 6(c) shows the significant decrease of impact force. The $M I F, P I F_{\mathrm{E}}$ and LMs mass of the designed TRM are $45.47 \mathrm{kN}, 77.32 \mathrm{kN}$, and $1.54 \mathrm{~kg}$, respectively. The better performance of the designed TRM with high impact force mitigation and lightweight LMs is achieved.

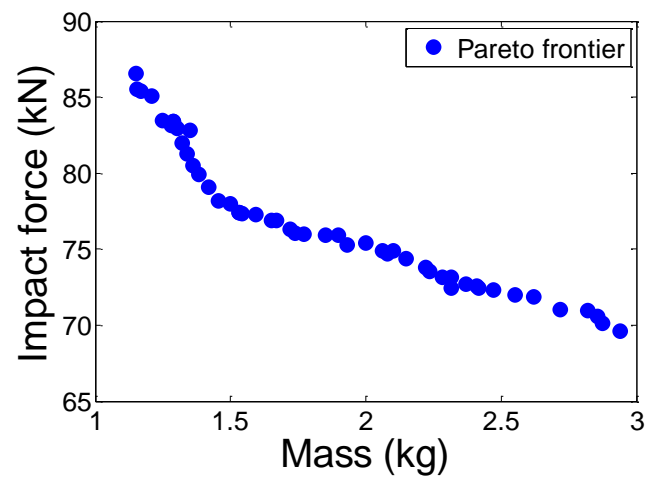

Fig. 8. Pareto frontier solutions of impact force against LMs mass.

Table 2 Comparison of Pareto-optimal solutions

\begin{tabular}{cccccc}
\hline Design variables & $\begin{array}{c}\text { 30-TRM: }(1.184,2.665, \\
\text { 4.737)*30 kN/mm }\end{array}$ & Option 1 & Option 2 & Option 3 & Option 4 \\
\hline$k_{2}(\mathrm{kN} / \mathrm{mm})$ & 1.184 & 3.395 & 2.494 & 1.373 & 0.998 \\
$k_{3}(\mathrm{kN} / \mathrm{mm})$ & 2.665 & 3.625 & 1.314 & 1.852 & 1.039 \\
$k_{4}(\mathrm{kN} / \mathrm{mm})$ & 4.737 & 4.775 & 3.741 & 3.696 & 3.642 \\
$m_{2}(\mathrm{~kg})$ & 0.03 & 0.0347 & 0.0180 & 0.0159 & 0.0100 \\
$m_{3}(\mathrm{~kg})$ & 0.03 & 0.0436 & 0.0411 & 0.0177 & 0.0154 \\
\hline
\end{tabular}




\begin{tabular}{cccccc}
\hline$m_{4}(\mathrm{~kg})$ & 0.03 & 0.0300 & 0.0187 & 0.0265 & 0.0124 \\
$S F M(\mathrm{kN} / \mathrm{kg})$ & 14.33 & 18.31 & 23.19 & 29.52 & 32.41 \\
$M I F(\mathrm{kN})$ & 38.70 & 52.32 & 48.22 & 45.47 & 37.36 \\
$P I F_{\mathrm{E}}(\mathrm{kN})$ & 84.18 & 70.57 & 74.67 & 77.32 & 85.53 \\
$M(\mathrm{~kg})$ & 2.70 & 2.86 & 2.08 & 1.54 & 1.15 \\
\hline
\end{tabular}

\section{The trolley crash model}

To further study the mitigation effect of LMs, we apply the TRM on the trolley crash condition to enhance the vehicle crashworthiness. For the vehicle crash condition, the vehicle acceleration has a great effect on the safety of passengers. The large peak acceleration may cause the serious injuries or even death of passengers in the collision. Therefore, the relatively small peak acceleration is urgently needed in the design of the vehicle crash. As we know, a linear relationship between the body acceleration and the impact force exists based on the Newton's second law. Therefore, in this section, we apply the LMs to reduce the peak acceleration.

Impact analysis is performed with one trolley model and two impact beam structures. As shown in Figs. 9 (a) and (b), the trolley with the weight of $500 \mathrm{~kg}$ impacts the rigid wall with the initial speed of $50 \mathrm{~km} / \mathrm{h}$. Two TRMs are installed in the beams. The material of the beams is dual-phase steel HC340/590DP that Young's modulus, Poisson's ratio and density are $21000 \mathrm{Mpa}, 0.3$ and $7.85 \mathrm{e}-6 \mathrm{~kg} / \mathrm{mm}^{3}$, and their thickness is $2.2 \mathrm{~mm}$. The effective stress and effective strain curve of this dual-phase steel is shown in Figs. 9 (c). The entire LMs system contains 784 unit cells. The parameters of $k_{2}, k_{3}, k_{4}, m_{2}, m_{3}$ and $m_{4}$ of the LMs model are $1.184 \mathrm{kN} / \mathrm{mm}, 2.665$ $\mathrm{kN} / \mathrm{mm}, 4.737 \mathrm{kN} / \mathrm{mm}, 0.02 \mathrm{~kg}, 0.02 \mathrm{~kg}$ and $0.02 \mathrm{~kg}$. Fig. 10 (b) and Table 4 show the results of this initial TRM model. From Fig. 10 (b), it is clearly observed that a 
significant drop of acceleration appears at 0 2 ms compared with the original trolley model. The drop of acceleration is from $187.91 \mathrm{~g}$ to $127.66 \mathrm{~g}$. The high performance of TRM in the improvement of crashworthiness is clearly proved.

Next, the multi-objective optimization analysis is carried out to reduce the mass of LMs. The ranges of each parameter are shown in Table 3. Minimization of the peak acceleration of the trolley and the LMs mass are set as the targets. Multi-objective optimization model is built by combining the parameterized model and the NSGA-II. The whole optimization process of the trolley model is the same as the above thin-walled structure crash model.
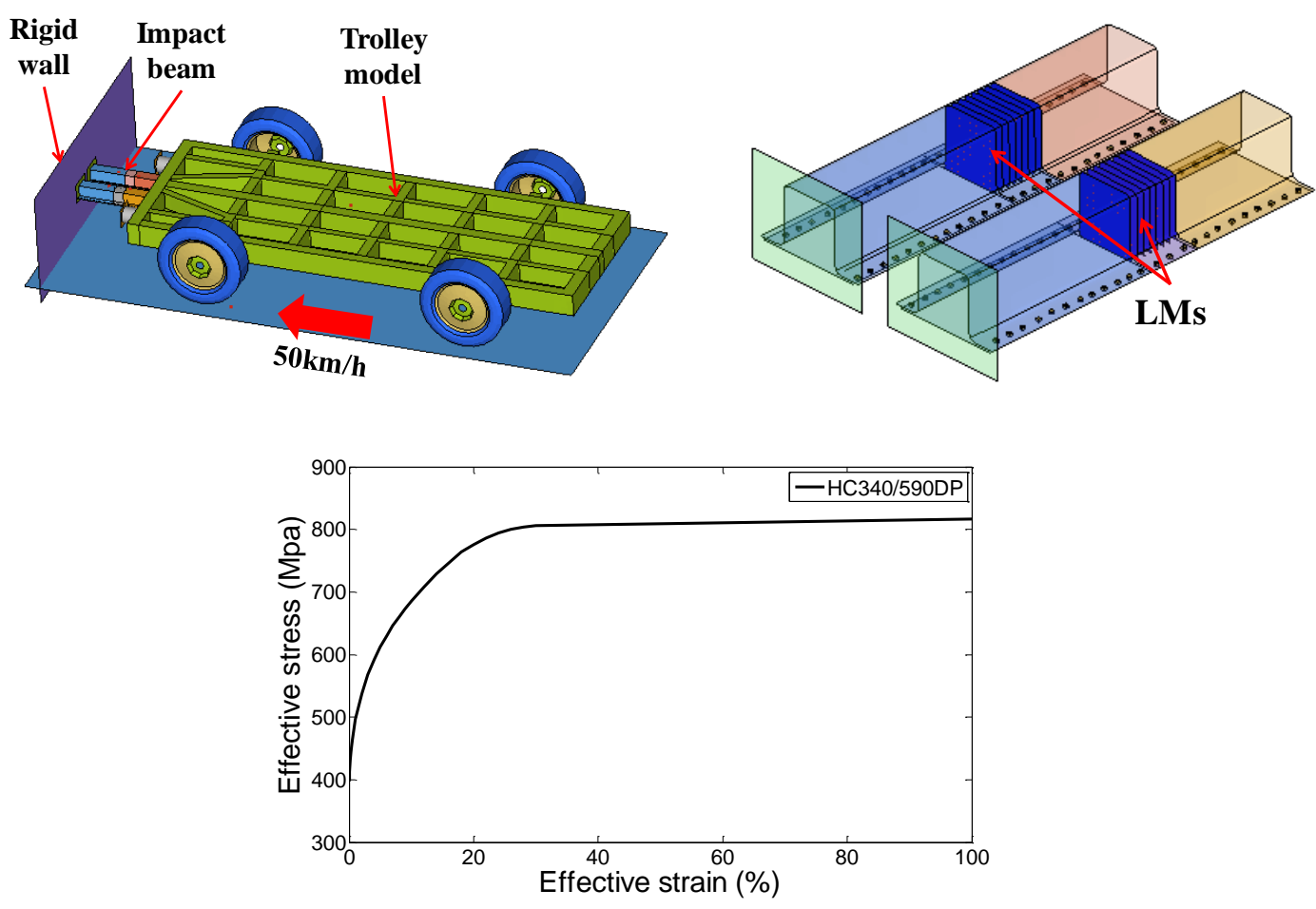

Fig. 9. (a) The trolley crash model, (b) The impact beam with LMs, (c) The effective stress and effective strain curve of dual-phase steel HC340/590DP.

Table 3. The initial value and range of each variable

\begin{tabular}{cccc}
\hline Design variable & Initial value & Lower & Upper \\
\hline$k_{2}(\mathrm{kN} / \mathrm{mm})$ & 0.790 & 0.079 & 8.39 \\
$k_{3}(\mathrm{kN} / \mathrm{mm})$ & 1.776 & 0.079 & 8.39 \\
\hline
\end{tabular}




\begin{tabular}{cccc}
\hline$k_{4}(\mathrm{kN} / \mathrm{mm})$ & 3.158 & 0.202 & 12.08 \\
$m_{2}(\mathrm{~kg})$ & 0.02 & 0.008 & 0.034 \\
$m_{3}(\mathrm{~kg})$ & 0.02 & 0.008 & 0.034 \\
$m_{4}(\mathrm{~kg})$ & 0.02 & 0.008 & 0.034 \\
\hline
\end{tabular}

Fifty feasible solutions are shown in Fig. 10 (a) and an optimum solution is selected for its small peak acceleration and lightweight LMs. Fig. 10 and Table 4 illustrate that the peak acceleration of the designed trolley model is reduced from $187.91 \mathrm{~g}$ to $126.03 \mathrm{~g}$ compared with the original model, which decrease ratio is $32.93 \%$. In addition, the mass of the designed TRM also drops from $47.00 \mathrm{~kg}$ to 36.26 $\mathrm{kg}$. From Table 5, the detailed parameters of the designed TRM are $k_{2}=1.07 \mathrm{kN} / \mathrm{mm}$, $k_{3}=4.77 \mathrm{kN} / \mathrm{mm}, k_{4}=5.54 \mathrm{kN} / \mathrm{mm}, m_{2}=0.014 \mathrm{~kg}, m_{3}=0.009 \mathrm{~kg}$ and $m_{4}=0.012 \mathrm{~kg}$. It is obvious that the performance of the designed TRM is better than the initial TRM. We can achieve the excellent performance of vehicle crashworthiness by simply adding the designed TRM to the impact beams of the trolley model.
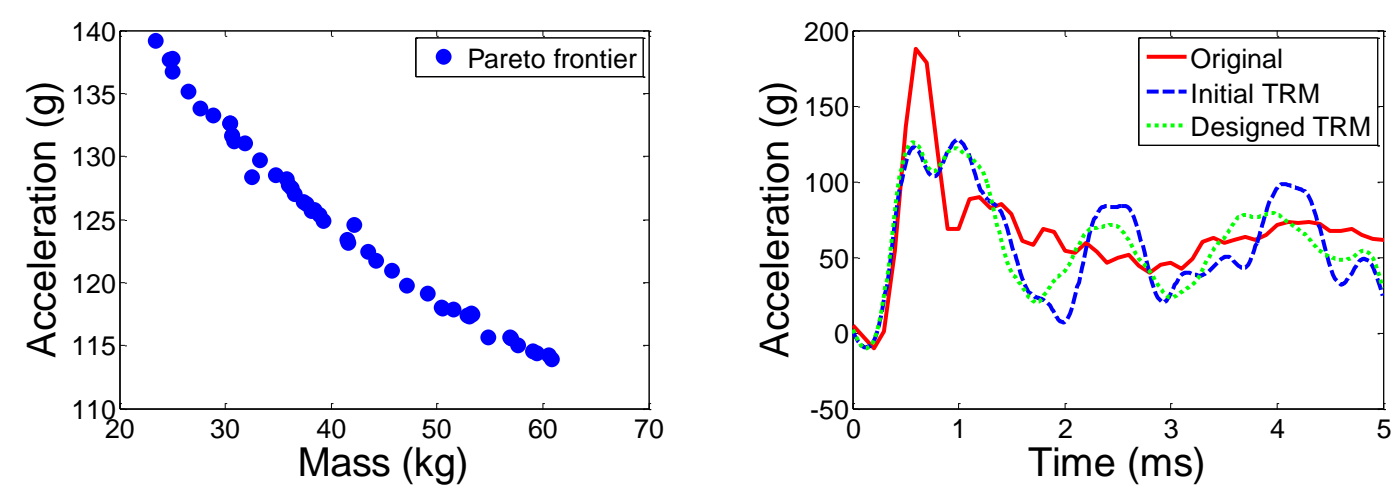

Fig. 10. (a) Pareto frontier of trolley acceleration against LMs mass, (b) Acceleration response of different trolley models.

Table 4. Numerical results of the initial TRM and the designed TRM

\begin{tabular}{cccc}
\hline & Peak acceleration $(\mathrm{g})$ & Decrease ratio $(\%)$ & Mass $(\mathrm{kg})$ \\
\hline Original & 187.91 & - & - \\
Initial TRM & 127.66 & 32.00 & 47.00 \\
Designed TRM & 126.03 & 32.93 & 36.26 \\
\hline
\end{tabular}

Table 5. The optimum combination of design parameters 


\begin{tabular}{cccccc}
\hline$k_{2}(\mathrm{kN} / \mathrm{mm})$ & $k_{3}(\mathrm{kN} / \mathrm{mm})$ & $k_{4}(\mathrm{kN} / \mathrm{mm})$ & $m_{2}(\mathrm{~kg})$ & $m_{3}(\mathrm{~kg})$ & $m_{4}(\mathrm{~kg})$ \\
\hline 1.07 & 4.77 & 5.54 & 0.014 & 0.009 & 0.012 \\
\hline
\end{tabular}

\section{Conclusion}

In this paper, the LMs are applied to achieve the impact force mitigation, and we propose a newly designed three-resonator metamaterial (TRM) to enhance the attenuation effect. Theoretical analysis shows its wide negative effective mass frequency regions and wide stopbands. Compared with the single-resonator metamaterial (SRM) and dual-resonator metamaterial (DRM), the advantages of TRM are more obvious in manipulating the impact stress wave. Numerical results of impact wave model and thin-walled column crash model demonstrate its superior performance compared with SRM and DRM in terms of high SFM value. Furthermore, multi-objective optimization analyses are carried out to design the TRM, and great improvement of these crash models with high impact force mitigation and light LMs mass is achieved.

\section{References}

1. Shima A, Takayama K, Tomita Y, Ohsawa N. Mechanism of impact pressure generation from spark-generated bubble collapse near a wall. Aiaa Journal. 1983;21(21):55-59.

2. Lu G, Yu T. - Energy Absorption of Structures and Materials. Energy Absorption of Structures \& Materials. 2003:385-400.

3. Chen W. Experimental and numerical study on bending collapse of aluminum foam-filled hat profiles. International Journal of Solids \& Structures. 2001;38(44):7919-7944.

4. Guo L, Yu J. Dynamic bending response of double cylindrical tubes filled with aluminum foam. International Journal of Impact Engineering. 2011;38(2-3):85-94.

5. Li Z, Zheng Z, Yu J, Guo L. Crashworthiness of foam-filled thin-walled circular tubes under dynamic bending. Materials \& Design. 2013;52(24):1058-1064.

6. Santosa S, Wierzbicki T. Effect of an ultralight metal filler on the bending collapse behavior of thin-walled prismatic columns. International Journal of Mechanical Sciences. 1999;41(8):995-1019.

7. Zhou G, Ma ZD, Gu J, Li G, Cheng A, Zhang W. Design optimization of a NPR structure based on HAM optimization method. Structural \& Multidisciplinary Optimization. 2016;53(3):1-9.

8. Fang N, Xi D, Xu J, Ambati M, Srituravanich W, Sun C, et al. Ultrasonic metamaterials with negative modulus. Nature materials. 2006;5(6):452-456. 
9. Fok L, Zhang X. Negative acoustic index metamaterial. Physical Review B. 2011;83(21):214304.

10. Liang Z, Li J. Extreme acoustic metamaterial by coiling up space. Physical review letters. 2012;108(11):114301.

11. He ZC, Li E, Wang G, Li GY, Xia Z. Development of an efficient algorithm to analyze the elastic wave in acoustic metamaterials. Acta Mechanica. 2016;227(10):3015-3030.

12. Li E, He ZC, Wang G. An exact solution to compute the band gap in phononic crystals. Computational Materials Science. 2016;122:72-85.

13. He ZC, Xiao X, Li E. Design for structural vibration suppression in laminate acoustic metamaterials. Composites Part B: Engineering. 2017;131:237-252.

14. Li E, He ZC, Hu JY, Long XY. Volumetric locking issue with uncertainty in the design of locally resonant acoustic metamaterials. Comput Method Appl M. 2017;324:128-148.

15. Li E, He ZC, Wang G, Liu GR. An ultra-accurate numerical method in the design of liquid phononic crystals with hard inclusion. Comput Mech. 2017; 60:1-14.

16. Pendry JB. Negative refraction makes a perfect lens. Physical review letters. 2000;85(18):3966.

17. Valentine J, Zhang S, Zentgraf T, Ulin-Avila E, Genov DA, Bartal G, et al. Three-dimensional optical metamaterial with a negative refractive index. nature. 2008;455(7211):376.

18. Dubey A, Shami T. Metamaterials in Electromagnetic Wave Absorbers. Defence Science Journal. 2012;62(4):261-268.

19. Shoukat S, Ahmed S, Ashraf MA, Syed AA, Naqvi QA. Scattering of electromagnetic plane wave from a chiral cylinder placed in chiral metamaterials. Journal of Electromagnetic Waves \& Applications. 2013;27(9):1127-1135.

20. Liu Z, Zhang X, Mao Y, Zhu Y, Yang Z, Chan CT, et al. Locally resonant sonic materials. Science. 2000;289(5485):1734-1736.

21. Cselyuszka N, Sečujski M, Crnojević-Bengin V. Novel negative mass density resonant metamaterial unit cell. Physics Letters A. 2015;379(1):33-36.

22. Assouar B, Oudich M, Zhou X. Acoustic metamaterials for sound mitigation. Comptes Rendus Physique. 2016;17(5):524-532.

23. Chen JS, Sharma B, Sun CT. Dynamic behaviour of sandwich structure containing spring-mass resonators. Composite Structures. 2011;93(8):2120-2125.

24. Zhu R, Huang $H$, Huang $G$, Sun $C$. Microstructure continuum modeling of an elastic metamaterial. International Journal of Engineering Science. 2011;49(12):1477-1485.

25. Huang $\mathrm{H}$, Sun $\mathrm{C}$. Continuum modeling of a composite material with internal resonators. Mechanics of Materials. 2012;46:1-10.

26. Zhang $Y$, Wen J, Xiao $Y$, Wen $X$, Wang J. Theoretical investigation of the sound attenuation of membrane-type acoustic metamaterials. Physics Letters A. 2012;376(17):1489-1494.

27. Wang Y F, Wang Y S, Wang L. Two-dimensional ternary locally resonant phononic crystals with a comblike coating. 2013, 47(1):15502-15509.

28. Wang Y F, Wang Y S, Laude V. Wave propagation in two-dimensional viscoelastic metamaterials. Physical Review B, 2015, 92(10): 104110.

29. Wang Y F, Wang Y S, Zhang C. Two-dimensional locally resonant elastic metamaterials with chiral comb-like interlayers: Bandgap and simultaneously double negative properties. The Journal of the Acoustical Society of America, 2016, 139(6): 3311-3319.

30. Yao S, Zhou X, Hu G. Experimental study on negative effective mass in a 1D mass-spring system. New Journal of Physics. 2008;10(4):043020. 
31. Manimala JM, Huang HH, Sun C, Snyder R, Bland S. Dynamic load mitigation using negative effective mass structures. Engineering Structures. 2014;80:458-468.

32. Zhou J, Cheng Y, Zhang H, Huang G, Hu G. Experimental study on interaction between a positive mass and a negative effective mass through a mass-spring system. Theoretical and Applied Mechanics Letters. 2015;5(5):196-199.

33. Naify CJ, Chang CM, Mcknight G, Nutt S. Transmission loss of membrane-type acoustic metamaterials with coaxial ring masses. Journal of Applied Physics. 2011;110(12):751.

34. Pai PF, Peng H, Jiang S. Acoustic metamaterial beams based on multi-frequency vibration absorbers. International Journal of Mechanical Sciences. 2014;79(1):195-205.

35. Assouar B, Oudich M, Zhou X. Acoustic metamaterials for sound mitigation. Comptes rendus Physique. 2016;17(5):524-532.

36. Wang X. Dynamic behaviour of a metamaterial system with negative mass and modulus. International Journal of Solids and Structures. 2014;51(7):1534-1541.

37. Yao S, Zhou X, Hu G. Experimental study on negative effective mass in a 1D mass spring system. New Journal of Physics. 2008;10(10):043020.

38. Li J, Chan C. Double-negative acoustic metamaterial. Physical Review E. 2004;70(5):055602.

39. Liu X, Hu G, Huang G, Sun C. An elastic metamaterial with simultaneously negative mass density and bulk modulus. Applied physics letters. 2011;98(25):251907.

40. Zhai S, Chen H, Ding C, Zhao X. Double-negative acoustic metamaterial based on meta-molecule. Journal of Physics D Applied Physics. 2013;46(47):5105.

41. Huang $H$, Sun $C$. Wave attenuation mechanism in an acoustic metamaterial with negative effective mass density. New Journal of Physics. 2009;11(1):013003.

42. Tan KT, Huang $H$, Sun $C$. Optimizing the band gap of effective mass negativity in acoustic metamaterials. Applied Physics Letters. 2012;101(24):241902.

43. Tan KT, Huang $H$, Sun $C$. Blast-wave impact mitigation using negative effective mass density concept of elastic metamaterials. International Journal of Impact Engineering. 2014;64:20-29.

44. Yin H, Wen G, Liu Z, Qing Q. Crashworthiness optimization design for foam-filled multi-cell thin-walled structures. Thin-Walled Structures. 2014;75:8-17.

45. Yin H, Xiao Y, Wen G, Gan N, Chen C, Dai J. Multi-objective robust optimization of foam-filled bionic thin-walled structures. Thin-Walled Structures. 2016;109:332-343.

46. Tang Z, Liu S, Zhang Z. Energy absorption properties of non-convex multi-corner thin-walled columns. Thin-Walled Structures. 2012;51:112-120.

47. Deb K, Pratap A, Agarwal S, Meyarivan T. A fast and elitist multiobjective genetic algorithm: NSGA-II. IEEE transactions on evolutionary computation. 2002;6(2):182-197.

48. Deb K, Goel T, editors. Controlled elitist non-dominated sorting genetic algorithms for better convergence. Evolutionary multi-criterion optimization. 2001:67-81.

49. Wang Y Z, Li F M, Wang Y S. Active feedback control of elastic wave metamaterials. Journal of Intelligent Material Systems and Structures, 2017, 28(15): 2110-2116.

50. Wang Y Z, Li F M, Wang Y S. Influences of active control on elastic wave propagation in a weakly nonlinear phononic crystal with a monoatomic lattice chain. International Journal of Mechanical Sciences, 2016, 106: 357-362. 